\title{
cAMP- and cGMP-elevating agents inhibit GPlba-mediated aggregation but not GPlba-stimulated Syk activation in human platelets
}

Stephanie Makhoul', Katharina Trabold ${ }^{1}$, Stepan Gambaryan ${ }^{1,2}$, Stefan Tenzer ${ }^{3}$, Daniele Pillitteri ${ }^{4}$, Ulrich Walter ${ }^{1}$ and Kerstin Jurk ${ }^{1 *}$ (D)

\begin{abstract}
Background: The glycoprotein (GP) Ib-IX-V complex is a unique platelet plasma membrane receptor, which is essential for platelet adhesion and thrombus formation. GPIba, part of the GPIb-IX-V complex, has several physiological ligands such as von Willebrand factor (VWF), thrombospondin and distinct coagulation factors, which trigger platelet activation. Despite having an important role, intracellular GPIb-IX-V signaling and its regulation by other pathways are not well defined. Our aim was to establish the intracellular signaling response of selective GPIba activation in human platelets, in particular the role of the tyrosine kinase Syk and its regulation by CAMP/PKA and cGMP/PKG pathways, respectively. We addressed this using echicetin beads (EB), which selectively bind to GPIba and induce platelet aggregation.

Methods: Purified echicetin from snake Echis carinatus venom was validated by mass spectrometry. Washed human platelets were incubated with EB, in the presence or absence of echicetin monomers (EM), Src family kinase (SFK) inhibitors, Syk inhibitors and the CAMP- and CGMP-elevating agents iloprost and riociguat, respectively. Platelet aggregation was analyzed by light transmission aggregometry, protein phosphorylation by immunoblotting. Intracellular messengers inositolmonophosphate (InsP1) and $\mathrm{Ca}^{2+}{ }_{i}$ were measured by ELISA and Fluo-3 AM/FACS, respectively.

Results: EB-induced platelet aggregation was dependent on integrin $\alpha_{\| b} \beta_{3}$ and secondary mediators ADP and $\mathrm{TXA}_{2}$, and was antagonized by EM. EB stimulated Syk tyrosine phosphorylation at Y352, which was SFKdependent and Syk-independent, whereas Y525/526 phosphorylation was SFK-dependent and partially Sykdependent. Furthermore, phosphorylation of both Syk Y352 and $Y 525 / 526$ was completely integrin $a_{\| b} \beta_{3^{-}}$ independent but, in the case of Y525/526, was partially ADP/TXA Y352/ Y525/Y526 phosphorylation, led to the phosphorylation of direct substrates (LAT Y191, PLCY2 Y759) and additional targets (Akt S473). PKA/PKG pathways inhibited EB-induced platelet aggregation and Akt phosphorylation but, surprisingly, enhanced Syk and LAT/PLCY2 tyrosine phosphorylation. A similar PKA/PKG effect was confirmed with convulxin-/GPVI-stimulated platelets. EB-induced InsP1 accumulation/InsP3 production and $\mathrm{Ca}^{2+}$-release were Syk-dependent, but only partially inhibited by PKA/PKG pathways.

(Continued on next page)
\end{abstract}

\footnotetext{
* Correspondence: kerstin.jurk@unimedizin-mainz.de

${ }^{1}$ Center for Thrombosis and Hemostasis (CTH), University Medical Center

Mainz of the Johannes Gutenberg University Mainz, Mainz, Germany

Full list of author information is available at the end of the article
}

(c) The Author(s). 2019 Open Access This article is distributed under the terms of the Creative Commons Attribution 4.0 International License (http://creativecommons.org/licenses/by/4.0/), which permits unrestricted use, distribution, and reproduction in any medium, provided you give appropriate credit to the original author(s) and the source, provide a link to the Creative Commons license, and indicate if changes were made. The Creative Commons Public Domain Dedication waiver (http://creativecommons.org/publicdomain/zero/1.0/) applies to the data made available in this article, unless otherwise stated. 
(Continued from previous page)

Conclusion: EB and EM are specific agonists and antagonists, respectively, of GPlba-mediated Syk activation leading to platelet aggregation. The CAMP/PKA and cGMP/PKG pathways do not inhibit but enhance GPIba-/ GPVI-initiated, SFK-dependent Syk activation, but strongly inhibit further downstream responses including aggregation. These data establish an important intracellular regulatory network induced by GPIba.

Keywords: CAMP-dependent protein kinase, cGMP-dependent protein kinase, Glycoprotein receptor GPIb-IX, Platelet activation, Syk kinase

\section{Plain English summary}

As the smallest circulating blood cells, platelets are activated at sites of vascular injury resulting in adhesion, aggregation, and blood coagulation, thereby preventing major blood loss. Pathologically, platelet hypofunction and hyperfunction can result in life-threatening bleeding or thromboinflammatory disorders, respectively. Platelet activation responses with subsequent thrombus formation are tightly controlled by multiple factors, which promote or inhibit platelet activation via membrane receptors and their intracellular effector systems. An essential platelet receptor is the glycoprotein (GP) Ib-IX-V complex, and its subunit GPIb $\alpha$ ligates predominantly von Willebrand factor but also other adhesion proteins and distinct coagulation factors. This enables platelet recruitment to the vessel wall, aggregation and coagulation. Despite this important role, intracellular effects of GPIb-IX-V in platelets and their interaction with other signaling pathways are not well defined. Recently, we characterized the snake venom protein echicetin immobilized on polystyrene beads as a specific GPIb activator. With this tool, we now show that GPIb $\alpha$ causes the activation of the tyrosine kinase Syk in a Src family kinase dependent manner, which results in the generation of further intracellular messengers and ultimately platelet aggregation. Surprisingly, activation of the platelet inhibitory cAMP/PKA and cGMP/PKG pathways enhanced initial Syk phosphorylation/activation, but strongly inhibited GPIb $\alpha$-induced platelet aggregation, which is distal to Syk activation. These studies establish a new intracellular regulatory network triggered by the activation of GPIba in human platelets.

\section{Background}

Circulating platelets are essential for both physiological and pathological hemostasis and have important roles in inflammatory diseases and cancer [1-3]. Platelet activating, inhibitory and modulating factors fine tune platelet adhesion to the vessel wall. Physiologically, this fine-tuning prevents excessive bleeding due to vascular injuries, and also prevents or limits pathological thrombus formation / vessel occlusion at sites of injured blood vessels.
Two major groups of platelet activators such as soluble agonists [thrombin, ADP, thromboxane $\mathrm{A}_{2}\left(\mathrm{TxA}_{2}\right)$ ] and adhesion molecules [e.g. von Willebrand factor (vWF), collagen, fibrin, podoplanin] bind to and stimulate specific G-protein-coupled receptors (GPCRs), or cell membrane-spanning adhesion receptors, respectively. These receptors stimulate intracellular signaling pathways and various platelet responses leading to integrin activation (e.g. integrin $\alpha_{\mathrm{IIb}} \beta_{3}$ ), granule secretion, exposure of anionic phospholipids [4-6] and subsequently firm adhesion, aggregation, thrombin generation and thrombus formation. Conversely, elevation of platelet cAMP or cGMP by endothelial-derived prostacyclin $\left(\mathrm{PGI}_{2}\right)$ or nitric oxide (NO) inhibits via cAMPdependent (PKA) and/or cGMP-dependent (PKG) protein kinases, respectively, many of these platelet activation responses at several sites of the activation pathways [7-9]. Activation of Src-family kinases (SFK) and subsequent protein tyrosine phosphorylation, including membrane proteins containing the "immunoreceptor tyrosine-based activation motif (ITAM)", initiates receptor-mediated platelet activation via the GPVI/Fc $\gamma$ chain [10-13], integrin $\alpha_{\text {IIb }} \beta_{3} /$ Fc $\gamma$ RIIA, CLEC-2, and GPIb-V-IX $[5,14,15]$.

ITAM-mediated Syk activation, discovered in immune cells $[16,17]$, is now established in many mammalian cells including platelets $[5,11,18]$. For human platelets, the presence of two ITAM proteins have been described, Fc receptor $\gamma$-chain (FcR $\gamma$; gene: FCER1G) and a low affinity IgG receptor FcyRIIa (gene: FCGR2A) [19, 20]. In murine platelets only the FcR $\gamma$ has been described [21, 22]. Studies with platelets from FcR $\gamma$-deficient mice established that this protein is essential for GPVI expression and function [12]. Cytosolic Syk is activated by two distinct overlapping mechanisms designated as ITAMdependent or Y-phosphorylation-dependent [18, 23-25]. The Syk Y-phospho-sites Y348/ Y352 and Y525/ Y526 belong to 2 pairs within the interdomain linker and kinase domain, respectively. Syk activation is initiated when these Y-sites are phosphorylated by SFKs or when dually Y-phosphorylated ITAM-containing membrane proteins recruit the two Syk-SH2 domains followed by Syk autophosphorylation [23, 24]. Usually, Syk activation is 
analyzed by Syk tyrosine phosphorylation (pY352 and pY525/526) and as tyrosine phosphorylation of its substrates (LAT pY191, PLC 2 pY759). However, there are many additional proteins known to be directly phosphorylated by Syk [26-28]. Over the last years, hundreds of Syk targets have been identified in proteomic/phosphoproteomic studies and used to generate distinct Syk networks in cancer cells [28, 29], and many of these Syk targets are present in human platelets.

vWF and its major receptor, the glycoprotein Ib-V-IX (GPIb-IX-V) complex, are essential for platelet adhesion and initial thrombus formation at sites of vascular injury under arterial and venous shear conditions [20,30,31]. The GPIb-IX-V complex consists of 4 transmembrane proteins (GPIb $\alpha$, GPIbß, GPIX, GPV; stoichiometry of 2: 2:2:1). GPIb $\alpha$ is of special importance since it binds most of the extracellular GPIb-IX-V complex ligands (e.g. vWF, thrombin, FIX, FXII, TSP-1, Mac-1, P-selectin), but also multiple intracellular ligands required for platelet activation [20]. The central role of the GPIb-V-IX complex in hemostasis is demonstrated by the strong bleeding disorder observed with Bernard-Soulier syndrome (BSS) patients who lack the platelet GPIb-V-IX complex, validated in studies with mice deficient in GPIb $\alpha[20,31]$. However, there are still many open questions concerning intracellular GPIb signaling and its interaction with other pathways.

When immobilized on the subendothelium of the damaged vessel wall, or presented on activated endothelial cells, the multimeric plasma protein vWF binds via its A1 domain to GPIb $\alpha$ and induces receptor clustering leading to platelet signaling and moderate activation. Without vessel wall components, snake venom toxins such as ristocetin or botrocetin are required for these vWF effects. Multiple intracellular signaling pathways have been proposed to mediate the intracellular effects of GPIb $\alpha$ activation by vWF, the phosphatidylinositol 3kinase (PI3K)/ protein kinase B (Akt) pathway, cGMP/ $\mathrm{PKG}$, the mitogen-activated protein kinase (MAPK) pathways and the Fc $\gamma$ R-Syk/PLC 2 pathway [30, 32-36]. However, the exact mechanisms have not been fully elucidated and are controversially discussed [20, 33, 37]. GPIb-V-IX is not the only platelet vWF receptor since the integrin $\alpha_{\mathrm{II}} \beta_{3}$ also ligates vWF via recognition of the RGD-sequence within the C4 domain of vWF, thereby inducing also platelet activation, often together with GPIba. As vWF is not a selective ligand of GPIb $\alpha$, studies of GPIb $\alpha$-selective signaling are rare and have so far been confined to platelets, which adhere to immobilized recombinant dimeric vWF A1 domain or active vWF A1-domains expressed on COS-7 cells [19, 30, 34] that also has limitations. The possible reasons for the often discrepant data and results published for GPIb signaling in human platelets are well reviewed and include the use of various GPIb-V-IX complex ligands, cell types, and biochemical and functional read-out systems [20, 38]. Recently, we developed a GPIb $\alpha$-specific agonist, the C-type lectin snake venom protein, echicetin, coated on polystyrene beads [39]. Earlier studies showed that echicetin molecules cross-linked by plasma IgMk caused platelet agglutination and weak aggregation whereas echicetin monomers, when used as specific GPIb $\alpha$ ligands, competed with vWF and thrombin for binding to GPIb $\alpha$ and blocked ristocetin/vWF mediated platelet agglutination [40]. Using a transgenic mouse model, we recently reported that the extracellular GPIba-domain of murine platelets is essential for echicetin-bead-induced platelet aggregation [41]. In this model the extracellular domain of GPIb $\alpha$ is replaced by the human interleukin 4-receptor (IL4/GPIb $\alpha$ transgenic mice). EB induced aggregation of washed platelets from wildtype mice, but not of washed platelets expressing the IL4/GPIba mutant protein. These data showed that EB activation of not only human but also mouse platelets requires GPIba, the predominant receptor for most ligands of the GPIb-IX-V complex.

With these novel tools, echicetin beads (EB) and echicetin monomers (EM), we aimed to clarify whether selective activation of human platelet GPIb $\alpha$ has the capacity to induce activation of the tyrosine kinase Syk and whether these pathways are affected by two major platelet inhibitory pathways, cAMP/PKA and cGMP/ PKG. Our results establish a novel, surprising interaction of GPIbo- and PKA/PKG-affected pathways in human platelets.

\section{Materials and methods Materials}

Lyophilized snake venom of E. carinatus sochureki was from Latoxan, France. Lyophilized convulxin (isolated from Crotalus durissus terrificus) was from Enzo life sciences, Lausen, Switzerland. Human vWF isolated from plasma (Wilate ${ }^{\circ}$ ) was from Octapharma $\mathrm{GmbH}$, Langenfeld, Germany. Ristocetin was from Loxo GmbH, Dossenheim, Germany. Affinity chromatography column, protein A sepharose-4B column coated with rabbit polyclonal antibodies against echicetin was from Dr. Alexei Navdaev, Würzburg, Germany. Diethylaminoethyl (DEAE) resin matrix, Toyopearls $650 \mathrm{~S}$ was from Tosoh Bioscience $\mathrm{GmbH}$, Germany. The resin was packed in Tricorn 5/50 column from GE healthcare life sciences, Germany. Syk inhibitors, OXSI-2, 2,3-Dihydro-3-[(1-methyl- $1 H$-indol-3-yl)methylene]-2-oxo- $1 H$-indole-5-sul-

fonamide were purchased from Merck, Germany and PRT060318 (PRT-318), (2-(1R,2S)-2-aminocyclohexylamino)-4-(m-tolylamino)pyrimidine-5-carboxamide), was from Selleckchem, Germany. PP2 and its inactive analogue PP3 were from Abcam, England. Tirofiban 
(Aggrastat $^{\circ}$ ) was from Iroko Cardio LLC, USA. MRS2179 was from Viozol, Eching, Germany, ARC69931 from The Medicines Company, Parsippany, NJ, USA and SQ-29548, was from Cayman chemical, MI, USA. Wortmannin was purchased from Biozol, Eching, Germany. Iloprost (Ilomedine ${ }^{\circ}$ ) and riociguat were from Bayer, Germany. Bovine serum albumin (BSA) was from Capricorn Scientific GmbH, Germany. Clarity ${ }^{\mathrm{Tm}}$ Western ECL Substrate was from BioRad Laboratories, USA. Rabbit monoclonal antibodies against phosphorylated Syk Y525/526 and polyclonal antibodies against phosphorylated Syk Y352, LAT Y191, PLCY2 Y759, Akt S473, VASP S239, $\alpha$-actinin were from Cell Signaling Technologies, USA. Mouse monoclonal antibodies against Syk, PLCy 2 and Akt were from Santa Cruz Biotechnology, USA. Antibodies against $\beta$-actin were from Abcam, Engalnd. Anti- phosphotyrosine mouse antibodies were from Merck, Schwalbach, Germany. Secondary antibodies HRP-conjugated goat anti-rabbit and anti-mouse IgG were from BioRad Laboratories Hercules, USA. IPOne ELISA kit (96 wells) was from Cisbio, Codolet, France. Fluo-3, AM, intracellular cytoplasmic $\mathrm{Ca}^{2+}$ indicator was from Life Technologies, USA.

\section{Echicetin purification and echicetin-beads preparation}

Echicetin was purified from E. carinatus sochureki lyophilized venom by affinity chromatography followed by DEAE anion exchange chromatography and validated by mass spectrometry analysis. For affinity chromatography protein A sepharose- $4 \mathrm{~B}$ column coated with rabbit polyclonal antibodies directed against echicetin (generated by A. Navdaev) was used. Echicetin was eluted using 0.2 $\mathrm{M}$ acetate buffer $\mathrm{pH}$ 2.7. The eluent buffer was exchanged into $10 \mathrm{mM}$ Tris buffer $\mathrm{pH} 8.0$ (buffer A) and then applied to DEAE anion exchange column. Elution of echicetin was performed by a 0 to $1 \mathrm{M}$ gradient of $\mathrm{NaCl}$ in buffer $\mathrm{A}$, under a flow rate of $1 \mathrm{ml} / \mathrm{min}$. Fraction eluted at $120 \mathrm{mM} \mathrm{NaCl}$ consisted equally of $\alpha$ and $\beta$ subunit and was used in all the experiments. Silver staining and mass spectrometry analysis were performed in order to confirm the purity of echicetin. Echicetin beads (EB) were prepared as reported [39] and coated for all experiments used with $0.3 \mathrm{mg} / \mathrm{ml}$ echicetin.

\section{LC-MS/MS}

Samples from peak 1 and peak 2 were prepared under reducing conditions (by adding Laemmli buffer) then boiled at $95^{\circ} \mathrm{C}$ for $10 \mathrm{~min}$. Proteins of both peaks were separated by electrophoresis using 15\% SDS-PAGE gels. Gels were stained using InstantBlue ${ }^{\mathrm{m}}$. Bands were cut and digested using trypsin. Protein sequences were analyzed by mass spectrometry in the mass spectrometry core facility at the University Medical Center of the Johannes Gutenberg University, Mainz.

\section{Preparation of washed human platelets}

Venous blood was collected as citrated whole blood after informed consent from healthy volunteers who did not take any medication for at least 10 days before blood collection. Studies using human platelets from healthy volunteers and from a patient with Glanzmann thrombosthenia caused by a homozygous point mutation in ITGA2B c.621C > T; p.T176I [42, 43] were approved by the local ethics committees (Study No. 837.302.12; 25.07.12; FF109/2015). EGTA (2 mM final concentration) was added to the whole blood before centrifuging at $200 \mathrm{x} \mathrm{g}$ for $10 \mathrm{~min}$ at room temperature (RT) to get platelet-rich plasma (PRP). PRP was diluted 1:1 with CGS buffer (120 mM NaCl, $12.9 \mathrm{mM}$ Tri-Na-citrate, 30 $\mathrm{mM}$ glucose, $\mathrm{pH}$ 6.5) then centrifuged at $400 \mathrm{x}$ g for 10 $\mathrm{min}$ at RT. The platelet pellet was resuspended with Hepes buffer $(150 \mathrm{mM} \mathrm{NaCl}, 5 \mathrm{mM} \mathrm{KCl}, 1 \mathrm{mM} \mathrm{MgCl}$, $10 \mathrm{mM}$ Glucose, $10 \mathrm{mM}$ Hepes) $\mathrm{pH}$ 7.4. Agglutination followed by fibrinogen-dependent aggregation of washed human platelets was induced by adding human vWF $(2.5 \mu \mathrm{g} / \mathrm{ml}$ final concentration) plus ristocetin $(0.5 \mathrm{mg} / \mathrm{ml}$ final concentration) as previously described [41]. Residual platelet aggregation was calculated by subtraction of overall maximum light transmission in the absence of tirofiban minus maximum light transmission in the presence of tirofiban. Washed platelets were placed for 20 $\min$ at $37^{\circ} \mathrm{C}$ for resting. Human washed platelets $(3 \times$ $10^{8}$ platelets $/ \mathrm{ml}$ ) were pre-incubated at $37^{\circ} \mathrm{C}$ with different inhibitors: Syk inhibitors, OXSI-2 or PRT-318, or Src family kinase inhibitor PP2 or its inactive analogue PP3 or wortmannin. In addition, ADP and TxA2 receptors blockers were used (AR-C69931, MRS2179, SQ29548), cAMP-elevating agents iloprost and cGMPelevating soluble guanylate cyclase (sGC) stimulator riociguat, respectively. Platelet aggregation was triggered with $0.5 \%(\mathrm{v} / \mathrm{v})$ echicetin beads for 1,2 and 5 min under continuous stirring $(1000 \mathrm{rpm})$ at $37^{\circ} \mathrm{C}$ in an Apact $4 \mathrm{~S}$ Plus aggregometer (DiaSys Greiner, Flacht, Germany). Control samples mentioned as basal (in graphs) or time zero (in blots and graphs) refer to washed platelets placed under continuous stirring for $1 \mathrm{~min}$ in absence of any agonist.

\section{Western blot analysis}

Western blot samples were prepared by immediately adding Laemmli buffer to washed platelets inside the $\mathrm{cu}$ vettes and boiled at $95^{\circ} \mathrm{C}$ for $10 \mathrm{~min}$. Platelet proteins were separated by electrophoresis using 8\% SDS-PAGE gels then transferred to polyvinylidene difluoride (PVDF) membranes before blocking the membranes for one hour with 5\% BSA in TBS-T $(20 \mathrm{mM}$ Tris, $140 \mathrm{mM}$ $\mathrm{NaCl}, 0.1 \%$ Tween, $\mathrm{pH} 7.6)$ at room temperature. Membranes were incubated overnight under gentle shaking with 1:1000 diluted primary antibodies. Membranes were 
washed three times with TBS-T and incubated for two hours at RT with the appropriate secondary antibodies diluted 1:5000 in 5\% BSA. Membranes were washed again for 3 times with TBS-T before developing the blots by ECL detection. Control blots with total proteins (Syk, $\mathrm{PLC} \gamma 2$ and Akt) derive from same samples from the corresponding phosphorylated form. $\alpha$-actinin was also used as loading control for distinct p-Akt blots. Blots with p-LAT, p-VASP were stripped and reprobed with anti- $\beta$-actin antibody. Blots were cropped following the corresponding molecular weight of the protein of interest.

\section{Inositol monophosphate (InsP1) measurement}

The production of inositol triphosphate (InsP3) was measured by the level of the InsP1 accumulated in washed platelets using the IP-One ELISA kit according to the manufacturer's instructions. Human washed platelets adjusted to $3 \times 10^{8} / \mathrm{ml}$ in presence of $\mathrm{LiCl}(1 \mathrm{mM})$, inhibiting the degradation of InsP1 into myo-inositol. Washed platelets were activated by EB in the absence or presence of effectors, under stirring conditions. Platelets were lysed after $5 \mathrm{~min}$ of stimulation then centrifuged at $16,000 \times \mathrm{g}$ for $10 \mathrm{~min}$ at $4{ }^{\circ} \mathrm{C}$. Platelets lysates were incubated with InsP1- HRP conjugate and anti- InsP1 monoclonal antibody for $3 \mathrm{~h}$.

\section{Intracellular $\mathrm{Ca}^{2+}$-release measurement}

Human platelets were prepared as already mentioned above and adjusted to $3 \times 10^{8} / \mathrm{ml}$ in Hepes buffer. Washed platelets were then pre-incubated with a $\mathrm{Ca}^{2+}$ indicator dye, fluo- 3 acetoxymethyl (AM) esters $(5 \mu \mathrm{M})$ for $30 \mathrm{~min}$ at $37^{\circ} \mathrm{C}$. Intracellular $\mathrm{Ca}^{2+}$-release was monitored for 2 min by flow cytometry after stimulation with EB without additional supplementation of extracellular $\mathrm{Ca}^{2+}$.

\section{Statistical analysis}

Experiments were performed at least three times with at least three different healthy donors. The data were presented as mean \pm standard deviation (S.D.). Statistical analysis was performed using GraphPad Prism 7 for Windows (GraphPad Software, San Diego, CA). The 2tailed Student's t-test was used for comparison of two groups, one-way and two-way ANOVA and Tukey's multiple comparison tests were used, when appropriate, for comparison of more than two groups. $P<0.05$ was considered as significant.

\section{Results}

Echicetin coated polystyrene beads activate Syk tyrosine kinase and stimulate $\alpha_{\| b} \beta 3$ integrin-dependent platelet aggregation

Initial experiments with conventionally purified echicetin from the snake venom Echis carinatus sochureki produced variable effects on platelets and prompted us to modify the purification procedure (Additional file 1: Figure S1). The final DEAE column produced 2 major protein peaks, which were characterized by mass spectrometry. Only peak 1 contained pure echicetin heterodimer (with $\alpha+\beta$ subunits), whereas peak 2 contained primarily the $\beta$ subunit and some unidentified bands (Additional file 1: Figure S1A, B). Echicetin eluted in peak 1 was used for all experiments reported here with consistent results, both with echicetin-coated beads (EB) or as echicetin monomer ( $\alpha / \beta$ subunits) (EM).

In agreement with our earlier work [39], the $\alpha_{\text {IIb }} \beta 3$ integrin antagonist tirofiban completely inhibited EBinduced platelet aggregation of washed platelets. EM, which alone selectively bind to GPIb $\alpha$ without inducing platelet activation, completely inhibited EB-induced platelet aggregation in a competitive manner. As negative-control, BSA coated beads did not show any effect on platelet aggregation (Additional file 1: Figure S1C, D). We also tested whether EM affect platelet aggregation induced by other main platelet receptors. Echicetin did not affect the aggregation induced by collagen (Additional file 2: Figure S2A, B), ADP (Additional file 2: Figure $\mathrm{S} 2 \mathrm{C}, \mathrm{D}$ ), $\mathrm{TxA}_{2}$ (Additional file 2: Figure S2E, F), TRAP6 (Additional file 2: Figure S2G, H), thrombin at high concentration (Additional file 2: Figure S2I, J). A small inhibitory effect was observed with aggregation induced by a low concentration of thrombin (Additional file 2: Fig. S2K, L), which possibly reflects the thrombin binding site of GPIb $\alpha[20,44]$.

For the possible Syk activation by EB, we analyzed the phosphorylation kinetics of the Syk activation marker, tyrosine 525/526, which is located in the activation loop of the kinase domain [45]. Washed human platelets stimulated with EB showed increased, but transient Syk phosphorylation at Y525/526 in a time dependent manner. EB-induced Syk Y526 phosphorylation was confirmed by LC-MS/MS (data not shown). Additionally, we investigated the phosphorylation of Syk at Y352, which is located in the $\mathrm{SH} 2$ kinase linker domain and shows an important role in Syk activation [23, 46]. Furthermore, we also measured the tyrosine phosphorylation of direct Syk substrates, inker for activation of T-cells (LAT) and phospholipase $\mathrm{C}_{2} 2$ (PLCY2), as indicator of Syk kinase activity. LAT Y191 phosphorylation was detectable but weak whereas PLCY2 Y759 phosphorylation had a similar time course as Syk tyrosine phosphorylation. In contrast, one of Syk downstream effectors and important PI3K effector, Akt, showed a delayed phosphorylation at S473 (Fig. 1a, b), which was completely inhibited by the PI3K inhibitor wortmannin (Fig. 1c, d), showing PI3K-dependency of Akt phosphorylation in EBinduced GPIb $\alpha$ signaling. 

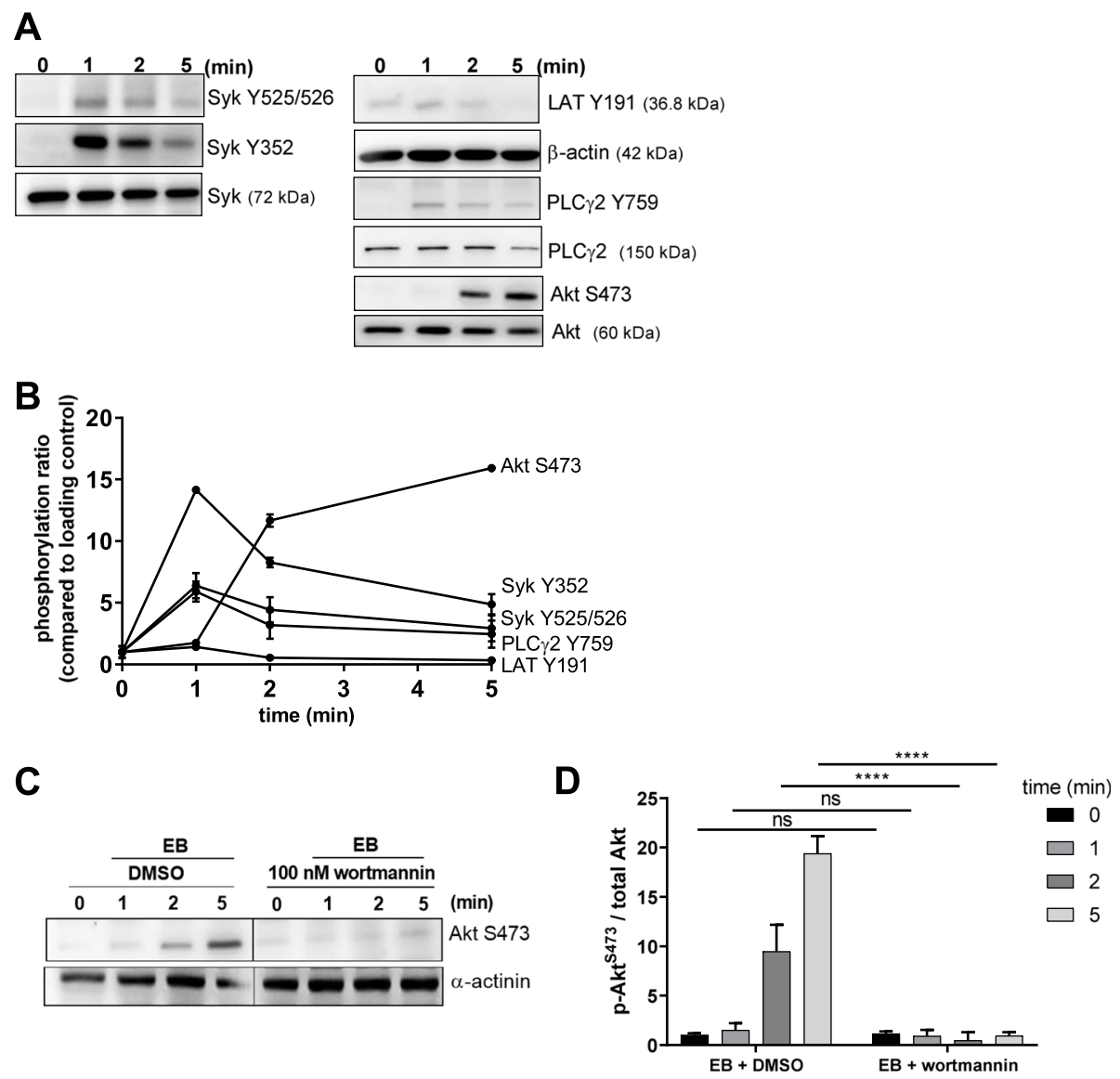

Fig. 1 Echicetin-coated polystyrene beads activate Syk tyrosine kinase and the PI3K-dependent serine/threonine kinase Akt. a Human washed platelets (WP) were stimulated under stirring conditions with EB. Platelet aggregation (see Fig. S1C, D) was stopped after 1, 2 or 5 min by adding Laemmli buffer. Tyrosine phosphorylation of Syk, LAT, PLCY2 and serine phosphorylation of Akt were analyzed by immunoblotting compared to the total protein respectively. $\mathbf{b}$ The kinetics of the phosphorylation patterns represent means \pm S.D. of 3 independent experiments with platelets from 3 healthy donors. c WP were pre-incubated for $5 \mathrm{~min}$ at $37^{\circ} \mathrm{C}$ with vehicle control (DMSO) or with the PI3K inhibitor wortmannin (100 nM final concentration) prior to stimulation with EB. $\mathbf{d}$ Quantitative data of Akt $\$ 473$ phosphorylation compared to the loading control a-actinin are represented as means \pm S.D. of 3 independent experiments with platelets from 3 healthy donors (Samples were loaded on the same gel; a black line was used to indicate that a group of samples not related to this data set was not shown); ns: not significant, ${ }^{* * *} p<0.0001$

\section{EB-induced Syk tyrosine phosphorylation requires src family kinases (SFKs)}

In order to study the role of SFKs in Syk activation, washed platelets were pre-incubated with a vehicle control (DMSO), with the SFK inhibitor PP2 and its inactive analogue PP3. EB-induced platelet aggregation was strongly inhibited only in the presence of PP2 (Additional file 3: Figure S3A, B). PP2 abolished timedependently $(0,15,60$ and $120 \mathrm{~s})$ EB-induced Syk phosphorylation at Y525/526 and Y352 (Fig. 2a, b, c). In contrast, neither Syk Y525/526 phosphorylation (Fig. 2d, e) nor Syk Y352 phosphorylation (Fig. 2d, f) were inhibited by PP3. Interestingly, dasatanib (100 $\mathrm{nM}$ ) also inhibited EB-induced platelet aggregation and EB-induced Syk Y352 and Y525/526 phosphorylation (data not shown). Dasatinib, originally developed for the treatment of chronic myelogenous leukemia
(CML), is not only an inhibitor of BCR-ABL but also a potent inhibitor of SFKs [47].

Syk inhibitors demonstrate an essential role of Syk in EBinduced platelet aggregation and a differential regulation of Syk Y525/526 and Y352 phosphorylation

To investigate the role of Syk in mediating EB- induced platelet activation, platelets were pre-incubated with two different, well-described Syk inhibitors, OXSI-2 or PRT318 [48-50]. Platelet aggregation was completely abolished by those two inhibitors (Fig. 3a, b), and global EBinduced tyrosine phosphorylation was partially inhibited (Additional file 4: Figure S4). Both Syk inhibitors caused a complete inhibition of EB-stimulated Syk Y525/526 phosphorylation, whereas Syk Y352 phosphorylation was not inhibited but prolonged (with OXSI-2) and prolonged/enhanced with PRT-318 (Fig. 4a, b, c), which clearly 
A

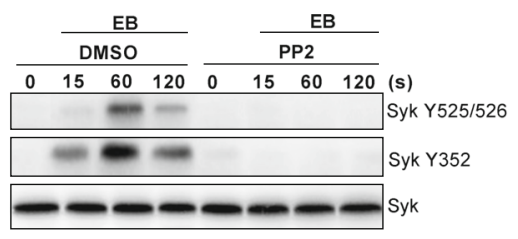

C

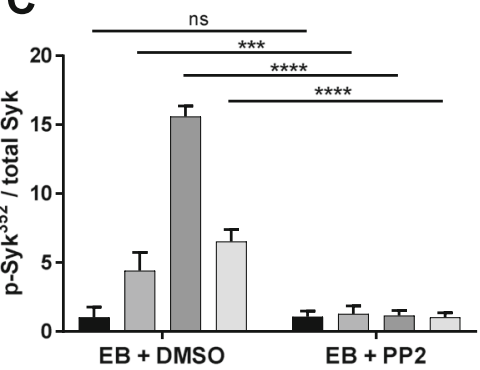

B

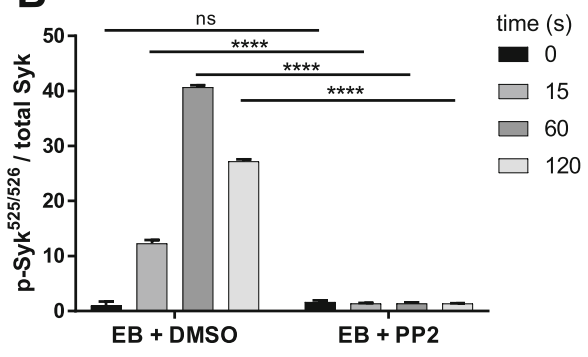

D

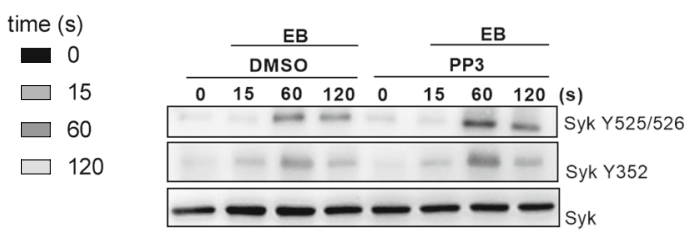

E
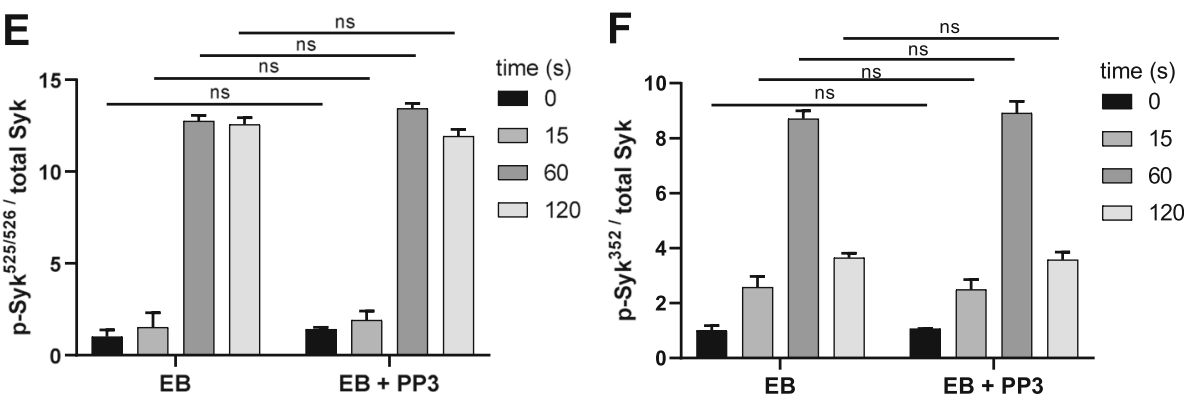

Fig. 2 EB-induced Syk tyrosine phosphorylation is dependent on src family kinases (SFKs). Human washed platelets (WP) were pre-incubated for 5 min with vehicle control (DMSO), the SFK inhibitor, PP2 (10 $\mu \mathrm{M})$ or with its inactive analogue, PP3 $(10 \mu \mathrm{M})$ prior to stimulation with EB. a, $\mathbf{d}$ Syk phosphorylation at Y525/526 and Y352 was analyzed at early time points (0, 15, 60 and 120 s). b, c, e, f Quantitative analysis of Syk Y525/526 and Y352 phosphorylation compared to total Syk. Data are means \pm S.D. from at least 3 independent experiments with platelets from at least 3 healthy donors; ns: not significant, ${ }^{* * *} p<0.001,{ }^{* * *} p<0.0001$

differed from the PP2 effects. These Syk inhibitors also strongly reduced EB-induced phosphorylation of PLC 2 at Y759, a direct Syk downstream target, and Akt at S473, a probably indirect Syk downstream target (Fig. $4 \mathrm{~d}$, e, f). For Akt we could show that the PI3K inhibitor wortmannin abolished EB-mediated Akt S473 phosphorylation (Fig. 1c, d) without inhibition of Syk tyrosine phosphorylation (data not shown). To compare these EB-mediated platelet effects with the classical platelet GPIb $\alpha$-agonist ristocetin, we analyzed vWF/ristocetininduced aggregation and Syk phosphorylation of washed human platelets in the presence of the Syk inhibitor PRT-318. PRT-318 inhibited partially vWF/ristocetin-induced platelet aggregation, but tirofiban-mediated inhibition of platelet aggregation was not further diminished by the PRT-318 compound (Additional file 4: Figure S4B, C), indicating that only vWF-mediated platelet aggregation but not agglutination was affected by the Syk inhibitor. Whereas vWF/ristocetin-induced Syk Y525/526 phosphorylation was clearly inhibited by PRT-318 (Additional file 4: Figure S4D), the effects were not markedly different in the presence of tirofiban. As observed for EB also vWF/ristocetin-induced Syk Y352 phoshorylation was not affected by PRT-318. To confirm that $\mathrm{vWF} /$ ristocetin-mediated Syk activity was inhibited by PRT-318, LAT was studied as direct substrate of Syk. vWF/ristocetin-induced LAT Y191 phosphorylation was similarly induced by vWF/ristocetin in the absence or presence of tirofiban and downregulated to basal levels in the presence of PRT-318 (Additional file 4: Figure S4, E). In addition, vWF/ristocetin-stimulated Akt S473 phosphorylation was downregulated by Syk inhibition, too. However, in the presence of tirofiban Akt phosphorylation was more diminished, indicating a role of integrin 

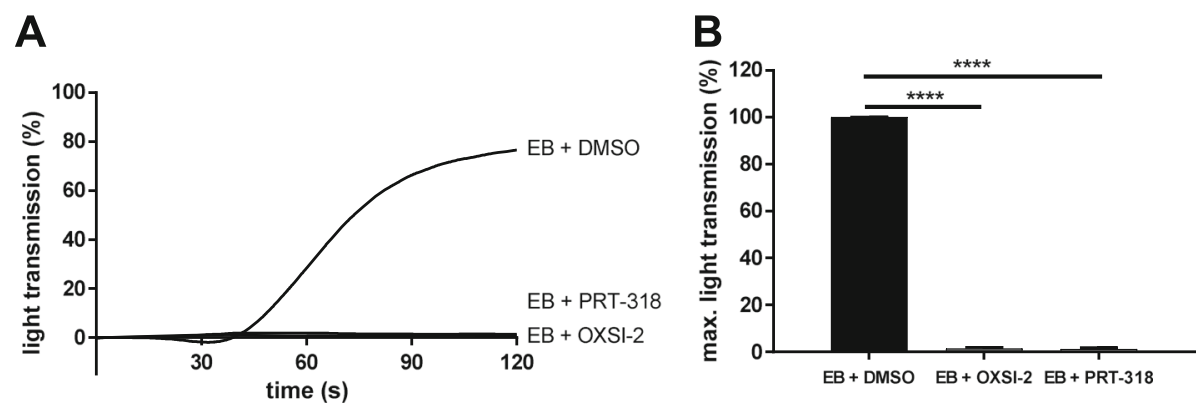

Fig. 3 Syk-inhibitors strongly block EB-induced platelet aggregation. Human washed platelets (WP) were pre-incubated with 2 different Syk inhibitors, OXSI-2 $(2 \mu \mathrm{M})$ and PRT-318 $(1 \mu \mathrm{M})$ for 5 min prior to stimulation with EB. a Representative curves showing the effect of Syk inhibitors on platelet aggregation and $\mathbf{b}$ the corresponding quantification of data as means \pm S.D. from 3 independent experiments with platelets from 3 healthy donors; ${ }^{* * *} p<0.0001$

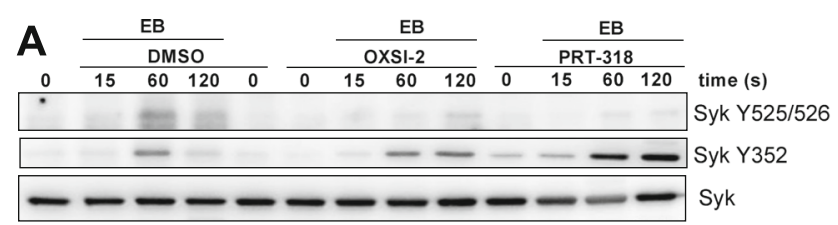

B
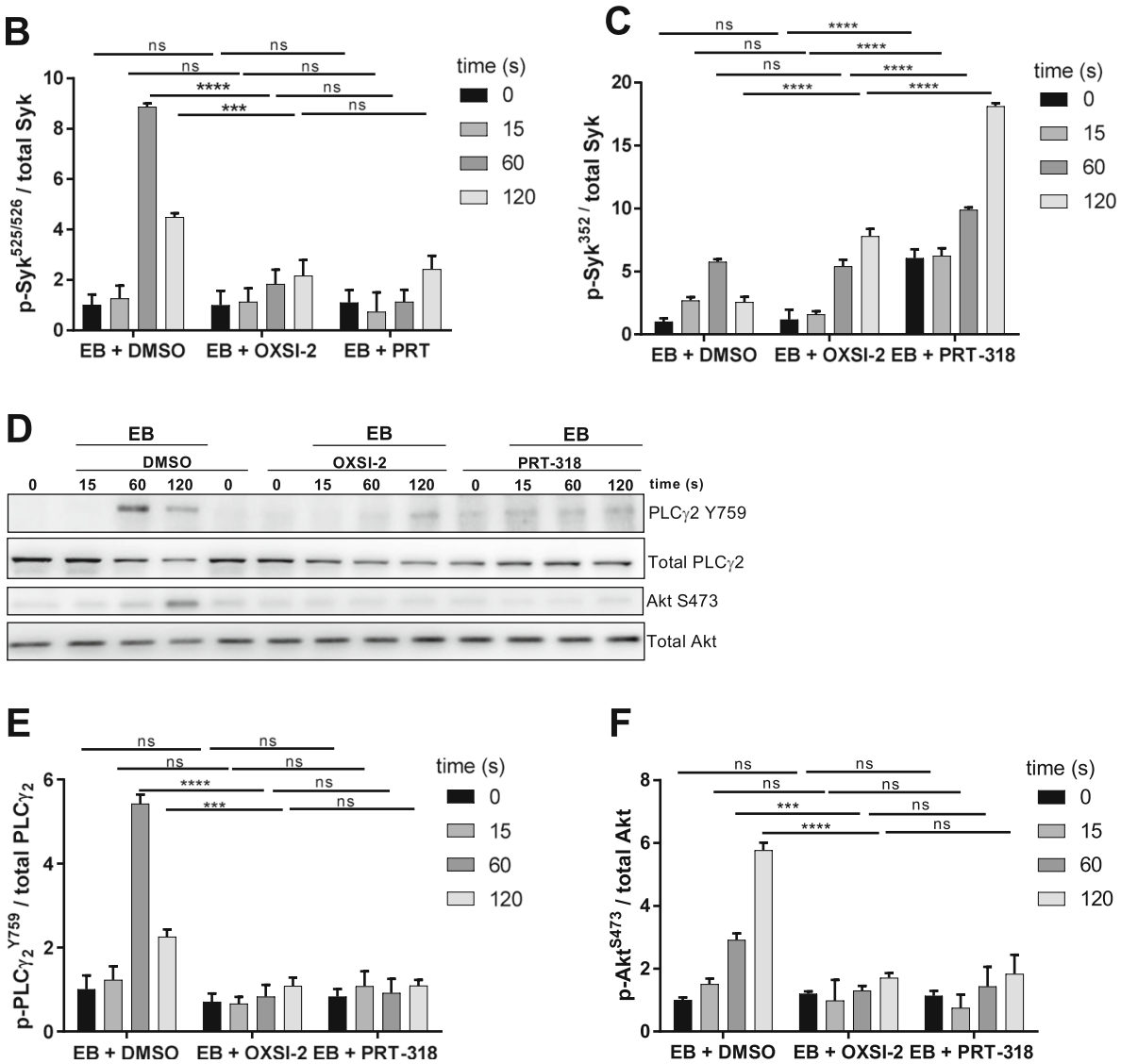

Fig. 4 Syk inhibitors differentially affect Syk tyrosine phosphorylation. Human washed platelets (WP) were treated as mentioned in Fig. 3. Samples were taken after 15, 60 and 120 s. a Representative blots of Syk phosphorylation on Y525/526 and Y352 and the corresponding quantification shown as ratio compared to the total Syk protein represented in $\mathbf{b}$ and $\mathbf{c}$ respectively. $\mathbf{d}$ Representative blots of Syk downstream effectors PLC 22 Y759 and Akt S473 and the corresponding quantification e and $\mathbf{f}$ shown as ratio compared to the total PLCY2 and Akt respectively. Data are means \pm S.D. from 3 independent experiments with platelets from 3 healthy donors; ns: not significant, ${ }^{* * *} p<0.001,{ }^{* * *} p<0.0001$ 
$\alpha_{\text {IIb }} \beta_{3}$ outside-in signaling in Akt S473 phosphorylation induced by $\mathrm{vWF} /$ ristocetin (Additional file 4: Figure S4E). These data demonstrate that Syk plays an important role in GPIb $\alpha$-mediated platelet activation induced by EB as well as by vWF/ristocetin.

\section{EB-induced platelet aggregation requires the secondary mediators ADP and $\mathrm{TxA}_{2}$}

Platelet adhesion receptor pathways variably require the activation-induced release of secondary mediators such as ADP and $\mathrm{TxA}_{2}$ for a full response [10]. Therefore, we evaluated the involvement of ADP and $\mathrm{TxA}_{2}$ in EBinduced platelet aggregation and phosphorylation responses by simultaneously blocking the $\mathrm{P}_{2} \mathrm{Y}_{12}, \mathrm{P}_{2} \mathrm{Y}_{1}$, and TP receptors using AR-C69931, MRS2179 and SQ29548, respectively. These conditions completely prevented platelet aggregation (Fig. 5a, b), whereas EBinduced Syk Y352 phosphorylation was not inhibited but instead prolonged at late time points (Fig. 5c, d, e). EBinduced Syk Y525/526 phosphorylation was partially inhibited by these compounds. In addition, phosphorylation of the direct downstream Syk effector, PLC $\gamma 2$ Y759, as well phosphorylation of Akt at S473 (Fig. 5f, g, h) were significantly inhibited.

\section{EB-mediated Syk activation does not require integrin $a_{1 \mathrm{lb}} \beta_{3}$ signaling}

Many signaling platelet pathways are affected by integrin out-side-in signaling, especially by the integrin $\alpha_{\mathrm{II}} \beta_{3}$. To address the possible role of the integrin $\alpha_{\mathrm{IIb}} \beta_{3}$ for GPIb $\alpha$-induced Syk activation we studied washed platelets from a patient with Glanzmann thrombasthenia (GT), which showed severe reduction of the major fibrinogen receptor $\alpha_{\mathrm{II}} \beta_{3}$ and which has been studied previously [51]. As expected, EB did not induce any aggregation response of platelets from a patient with GT, whereas platelets from a healthy control showed clear aggregation in response to EB, which was completely inhibited by the cAMP-elevating agent iloprost (Fig. 6a). EB induced the phosphorylation of Syk at both sites Y525/526 and Y352 in thromboasthenic as well as in control platelets (Fig. 6b). Furthermore, we investigated the effect of the integrin $\alpha_{\mathrm{IIb}} \beta_{3}$ inhibitor tirofiban on Syk activation with platelets from healthy controls. EBinduced Syk phosphorylation in platelets pre-incubated with tirofiban was not different when compared to nontreated control platelets. Also, the combination of iloprost and tirofiban did not differ in the phosphorylation pattern of Syk compared to platelets pre-incubated with iloprost alone (Fig. 6c, d, e, f). Additionally, tirofiban alone did not inhibit the phosphorylation of PLC $\gamma 2$ Y759. Only the combination, tirofiban plus iloprost, induced a partial inhibition in the phosphorylation pattern
(Fig. 6 g, h). The effects of iloprost alone on EB-induced Syk phosphorylation are presented next.

\section{cAMP/ cGMP elevation inhibits EB-induced platelet aggregation but not Syk activation, an effect also observed with convulxin-treated platelets}

We then evaluated possible effects of the cAMP/PKA and cGMP/PKG pathways on GPIb $\alpha$-mediated platelet signaling and aggregation. Previously, we had established conditions for specific cAMP/PKA- or cGMP/PKG-mediated phosphorylation of established PKA and/or PKG substrates (e.g. VASP S157, VASP S239, CALDAG GEF1 S587, phosphodiestersase PDE5A S102) by functional studies, immunoblotting and phosphoproteomic analysis using iloprost or riociguat $[8,52-54]$. Here, preincubation of washed platelets with increasing concentrations of iloprost (Additional file 5: Figure S5A) and riociguat (Additional file 5: Figure S5B) inhibited EBstimulated platelet aggregation in a dose dependent manner. For further studies, we used $2 \mathrm{nM}$ iloprost and $20 \mu \mathrm{M}$ riociguat (with established phosphoproteomic responses $[8,52]$ ), which caused a strong inhibition of EBstimulated platelet aggregation (Fig. 7a, b). Under these conditions, a consistently robust and stable phosphorylation of VASP S157 (substrate for PKA > PKG) and VASP S239 (substrate for PKG > PKA) was observed. Whereas VASP 239 phosphorylation was measured by phosphoantibodies, VASP S157 phosphorylation is indicated by the apparent shift of VASP from the $46 \mathrm{kDa}$ to the $50 \mathrm{kDa}$ form in SDS PAGE. Here, it is important to note that EB alone did not result in any VASP phosphorylation and that the observed iloprost or riociguatstimulated VASP phosphorylation was not downregulated by EB treatment (Additional file 5: Figure S5C, D). Furthermore, the PKG specific substrate phosphodiesterase PDE5A S102 was phosphorylated only in the riociguat-treated samples, but not in response to iloprost (data not shown). Overall, our present results show the selective and robust activation of PKA and PKG by iloprost and riociguat, respectively. Then, the effects of PKA or PKG pathways on the kinetics of the EBinduced Syk activation and phosphorylation of selected downstream effectors (LAT, PLC 2 and Akt) were investigated. In contrast to the total inhibition of EB-induced aggregation, iloprost and riociguat did not inhibit but enhanced/prolonged phosphorylation of the Syk activation marker Y525/526 (hyperphosphorylation), detectable at early and late time points of EB stimulation (Fig. $7 \mathrm{c}, \mathrm{d})$. With the regulatory site Y352, iloprost and riociguat moderately reduced phosphorylation initially (at 1 min EB stimulation) but then (2 min, $5 \mathrm{~min}$ ) did not inhibit but prolong Syk Y352 phosphorylation (Fig. 7c, e). Since the data indicate that the strong inhibitory pathways PKA/PKG do no prevent Syk activation under 

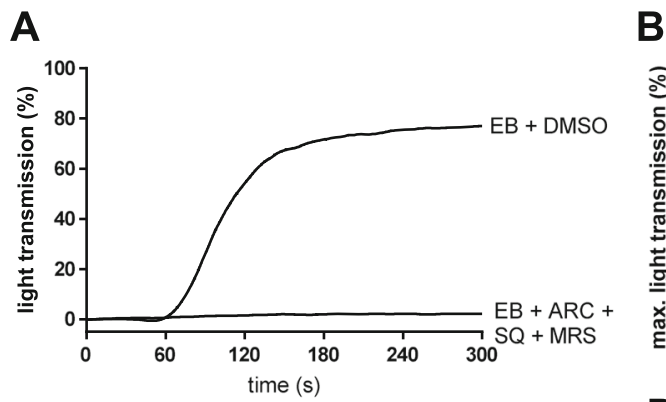

B
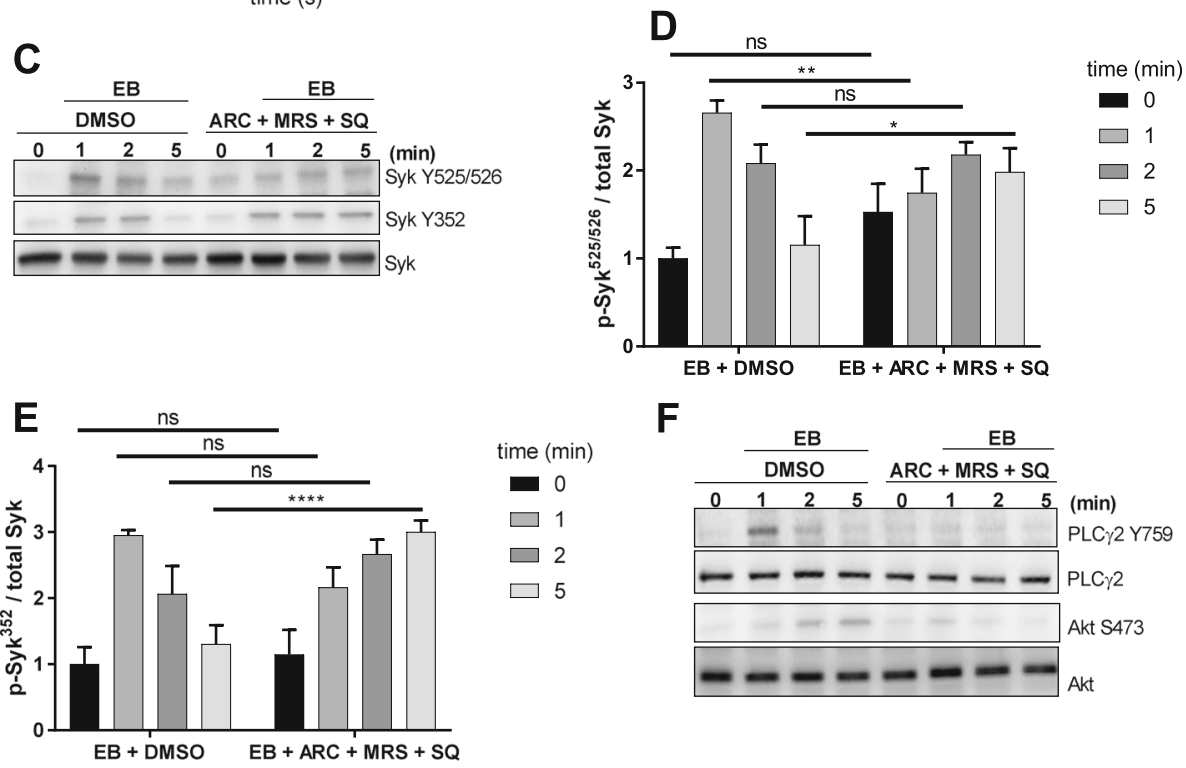

F

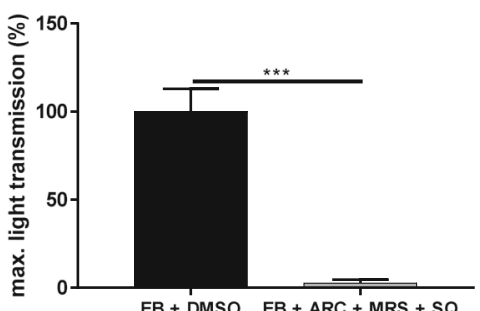

\section{D}

$\square 1$

$\square$

F

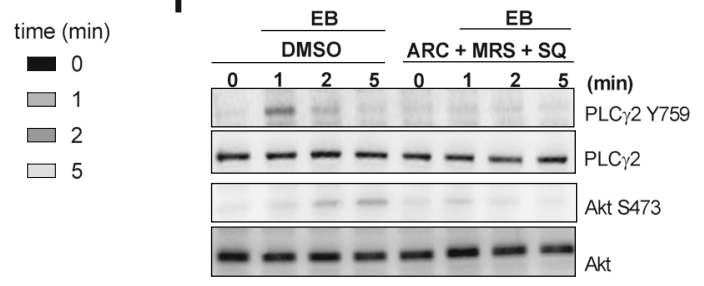

G

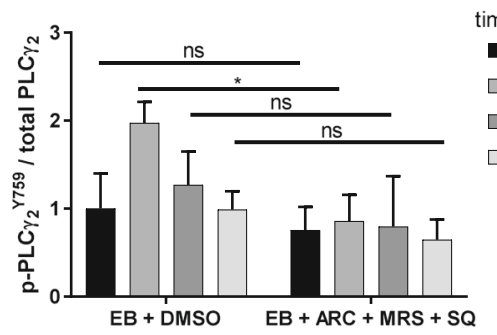

H

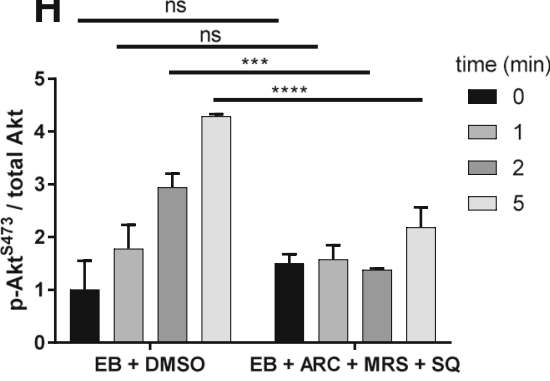

Fig. $5 \mathrm{ADP}$ and $\mathrm{TXA}_{2}$ regulate EB-induced platelet aggregation and Syk downstream signaling. Human washed platelets (WP) were pre-incubated

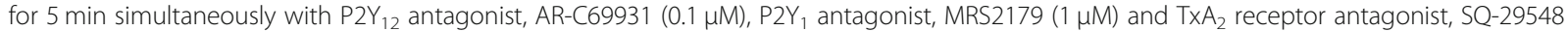
$(1 \mu \mathrm{M})$ prior to stimulation with EB. Samples were taken after 1, 2 and 5 min. a Representative curves of platelet aggregation mediated by EB in the presence of the vehicle control or the 3 inhibitors and the corresponding quantification are represented in $\mathbf{b}$. c Representative blots of Syk phosphorylation on Y525/526 and Y352 and the corresponding quantification shown as ratio compared to the total Syk protein represented in $\mathbf{d}$ and $\mathbf{e}$ respectively. $\mathbf{f}$ Representative blots of Syk downstream effectors PLCY2 Y759 and Akt S473 and the corresponding quantification $\mathbf{g}$ and $\mathbf{h}$ shown as ratio compared to the total PLCY2 and Akt, respectively. Data are presented as means \pm S.D. from 3 independent experiments with 3 healthy donors; ns: not significant, ${ }^{*} p<0.1,{ }^{* *} p<0.01,{ }^{* * *} p<0.001,{ }^{* * *} p<0.0001$

these conditions we sought to confirm this at the level of the Syk substrates LAT Y191 (Fig. 7c, f) and PLCY2 Y759 (Fig. 7c, g). Both Syk-mediated phosphorylation events were not inhibited but strongly enhanced/prolonged until $5 \mathrm{~min}$ of activation. In contrast, the EBinduced, delayed Akt phosphorylation at S473 was abolished by both iloprost and riociguat (Fig. 7c, h), which resembles the aggregation response.

Then we also used lower concentrations of iloprost (1 $\mathrm{nM})$ and riociguat $(10 \mu \mathrm{M})$, which partially inhibit EBinduced platelet aggregation, to test if the observed protein phosphorylation effects are still present compared 
A

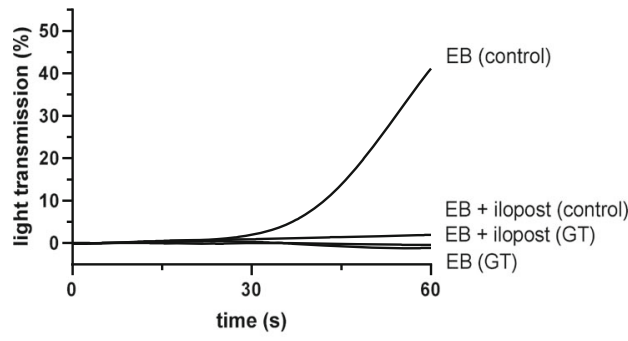

C

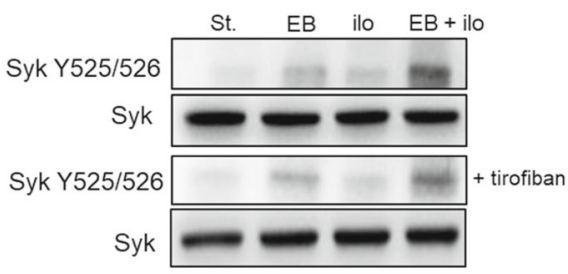

E

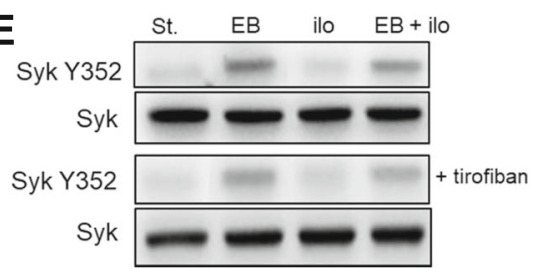

G

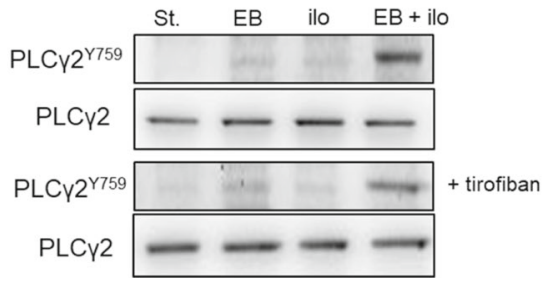

B

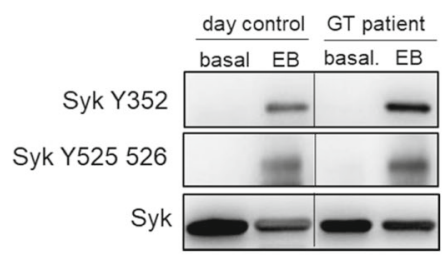

D
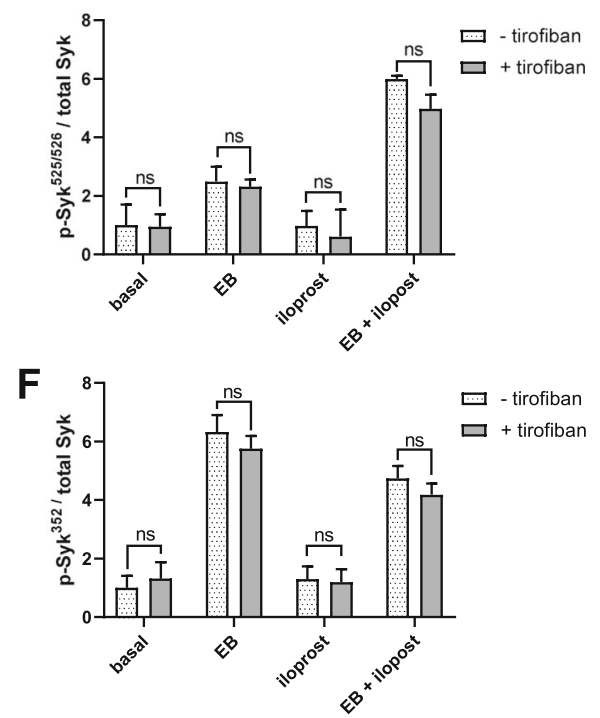

H

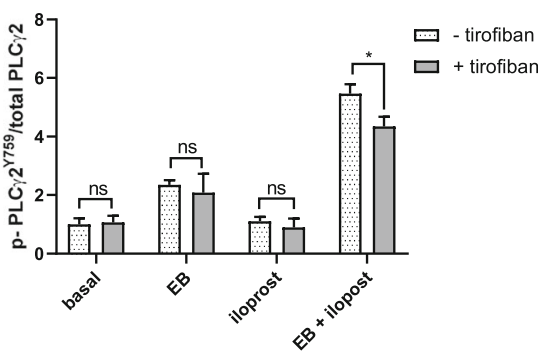

Fig. 6 EB-induced Syk activation is not dependent on integrin allb $\beta 3$ outside-in signaling in contrast to the overall platelet aggregation. a Washed platelets (WP) from a patient with Glanzmann thrombasthenia (GT) and from a healthy donor (day control) were pre-incubated at $37^{\circ} \mathrm{C}$ for $3 \mathrm{~min}$ in the presence or absence of $2 \mathrm{nM}$ iloprost prior to stimulation with EB. Platelet aggregation was monitored until $60 \mathrm{~s}$ using light transmission aggregometry under stirring conditions and then stopped using Laemmli buffer for immunoblotting. b Platelet aggregation was stopped after 60 s using Laemmli buffer and Syk Y525/526 and Y352 were analyzed by immunoblotting. WP from a healthy donor were preincubated with iloprost $(2 \mathrm{nM}, 3 \mathrm{~min})$ in the presence or absence of tirofiban $(1.25 \mu \mathrm{g} / \mathrm{ml}, 1 \mathrm{~min})$ prior to stimulation with EB. Platelet aggregation was stopped after 60 s using Laemmli buffer. Phosphorylation of c Syk Y525/526, e Syk Y352 and g PLCY2 Y759 were analyzed by western blot. Quantification of p-Syk $\mathbf{d}$ Y525/526 and $\mathbf{f}$ Y352 compared to total Syk and h PLCY2 Y759 compared to total PLCY2. Data are from 3 different experiments with platelets from 3 healthy volunteers and presented as means \pm S.D. ns: not significant, ${ }^{*} p<0.05$

to $2 \mathrm{nM}$ iloprost and $20 \mu \mathrm{M}$ riociguat, respectively. For $1 \mathrm{nM}$ iloprost (Additional file 6: Figure S6A-E) as well as for $10 \mu \mathrm{M}$ riociguat (Additional file 7: Figure S7A-E) we observed also increased and prolonged phosphorylation of Syk Y525/526, Syk Y325 and PLC 2 Y759 as well as diminished Akt S473 phosphorylation (Additional file 6:
Figure S6D, F and Additional file 7: Figure S7D, F), indicating similar effects of iloprost and riociguat on EBinduced platelet signaling.

The surprising differential effects of the inhibitory pathways (PKA/PKG) on EB-simulated platelet aggregation and Syk activation prompted us to study also a 

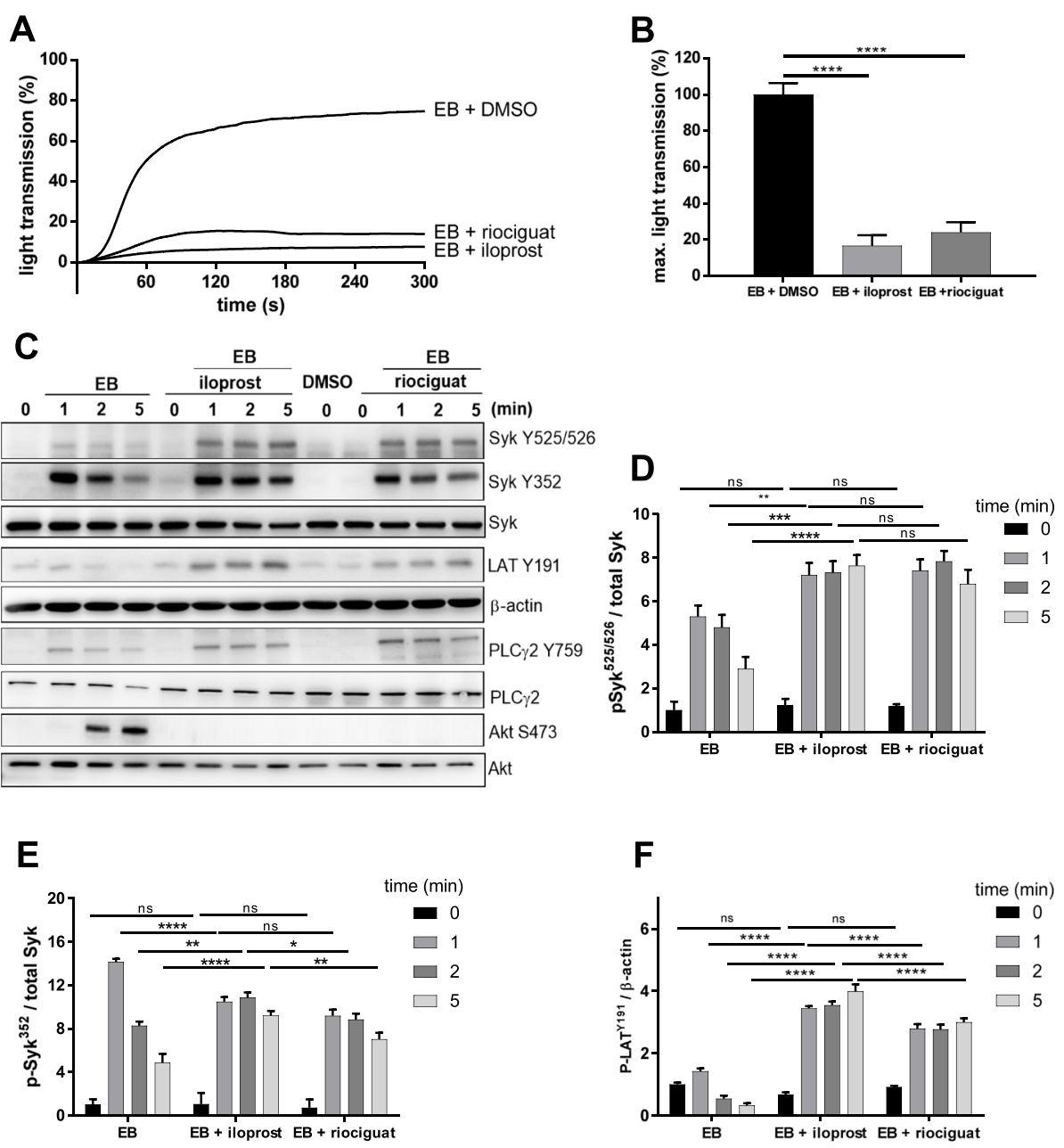

G

\section{H}
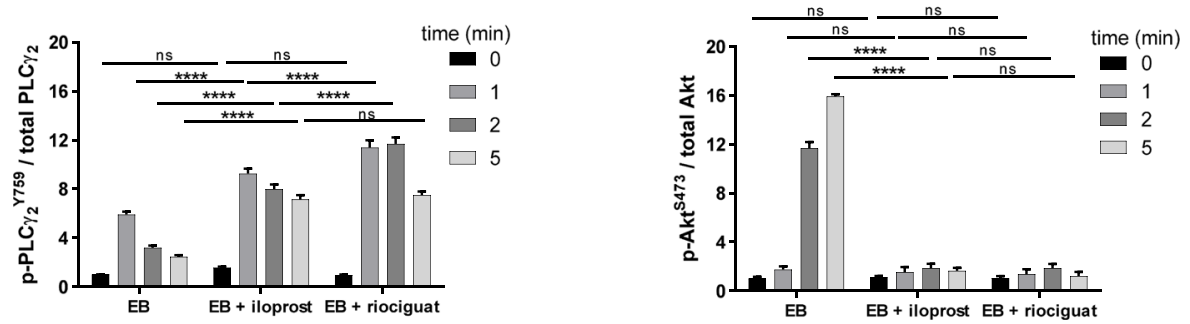

Fig. 7 lloprost and riociguat inhibit EB-induced platelet aggregation but do not inhibit tyrosine phosphorylation of Syk. Washed platelets (WP) were pre-incubated for 3 or 2 min with $2 \mathrm{nM}$ iloprost or $20 \mu \mathrm{M}$ riociguat respectively prior to stimulation with EB. a Aggregation curves and the corresponding quantitative data are shown in b. c Aggregation was stopped after 1, 2 or 5 min using Laemmli buffer. Syk Y525/526 and Y352, Syk downstream effectors LAT Y191, PLCY2 Y759 and Akt S473 were analyzed by western blot. d, e, f, $\mathbf{g}, \mathbf{h}$ Quantification of the phosphorylated proteins are represented as ratio compared to the total protein or compared to $\beta$-actin for LAT analysis. Results are presented of least 3 different experiments with platelets from at least 3 healthy volunteers, data are represented as means \pm S.D. ns: not significant, ${ }^{*} p<0.1,{ }^{* *} p<0.01$, ${ }^{* * *} p<0.001,{ }^{* * * *} p<0.0001$

classical mechanism of Syk activation in human platelets, the convulxin/GPVI pathway [10-12]. For the analysis of PKA/PKG effects on GPVI-mediated signaling pathways, we used $50 \mathrm{ng} / \mathrm{ml}$ convulxin to stimulate washed human platelets. Convulxin-stimulated platelet aggregation was completely inhibited by iloprost and riociguat (Additional file 8: Figure S8A, B). Then, convulxin-induced Syk phosphorylation in the presence of a vehicle control, iloprost or riociguat was analyzed. The Syk activation marker Y525/526 and the regulatory site Y352 were well 
but only transiently phosphorylated in response to convulxin (Additional file 8: Figure S8C). Strikingly, convulxinstimulated Syk Y525/526 phosphorylation was significantly increased/prolonged (hyperphosphorylation) by iloprost and riociguat compared to the vehicle control (Additional file 8: Figure S8C, D), which was supported by LC-MS/MS (data not shown). With Syk Y352 phosphorylation, there was little effect after $1 \mathrm{~min}$ of cvx stimulation, but at 2 and $5 \mathrm{~min}$ there was also significantly increased and prolonged phosphorylation in the presence of both inhibitory agents (Additional file 8: Figure S8C, E).

EB-stimulated intracellular InsP1 accumulation and $\mathrm{Ca}^{2+}$ release are only partially inhibited by iloprost and riociguat

In order to analyze a functional response in platelets directly after Syk-mediated PLC 22 phosphorylation/activation, we measured EB-induced InsP1 accumulation (in the presence of lithium) as marker of EB-mediated InsP3 production as reported [55]. We detected a clear 3-fold increase of InsP1 accumulation of EB-treated platelets, which was abolished by pre-incubation with EM (as control) and by the Syk inhibitor PRT-318 (Fig. 8a). Iloprost and riociguat only partially inhibited this EB-mediated InsP1 accumulation, which was more strongly reduced when ADP/TP receptors were blocked. The combination of these inhibitors did not produce additive effects (Fig. 8b). Also, pre-incubation of platelets with tirofiban did not reduce InsP1 accumulation, and the combination of iloprost with tirofiban showed the inhibition of EB-induced InsP1 production as with iloprost alone (Fig. 8b). Furthermore, we detected a significant increase of endogenous $\mathrm{Ca}^{2+}$-release upon platelet activation with $\mathrm{EB}$, which was abolished by PRT-318. However, iloprost and riociguat showed only partial inhibition of EB-induced $\mathrm{Ca}^{2+}$-release. In addition, EB-induced $\mathrm{Ca}^{2+}$-release showed to be independent from the integrin $\alpha_{\text {IIb }} \beta_{3}$ outside-in signaling as tirofiban had no significant effect (Fig. 8c).

\section{Discussion}

In this study we demonstrated that cAMP/PKA and cGMP/PKG pathways cause dichotomous regulation of

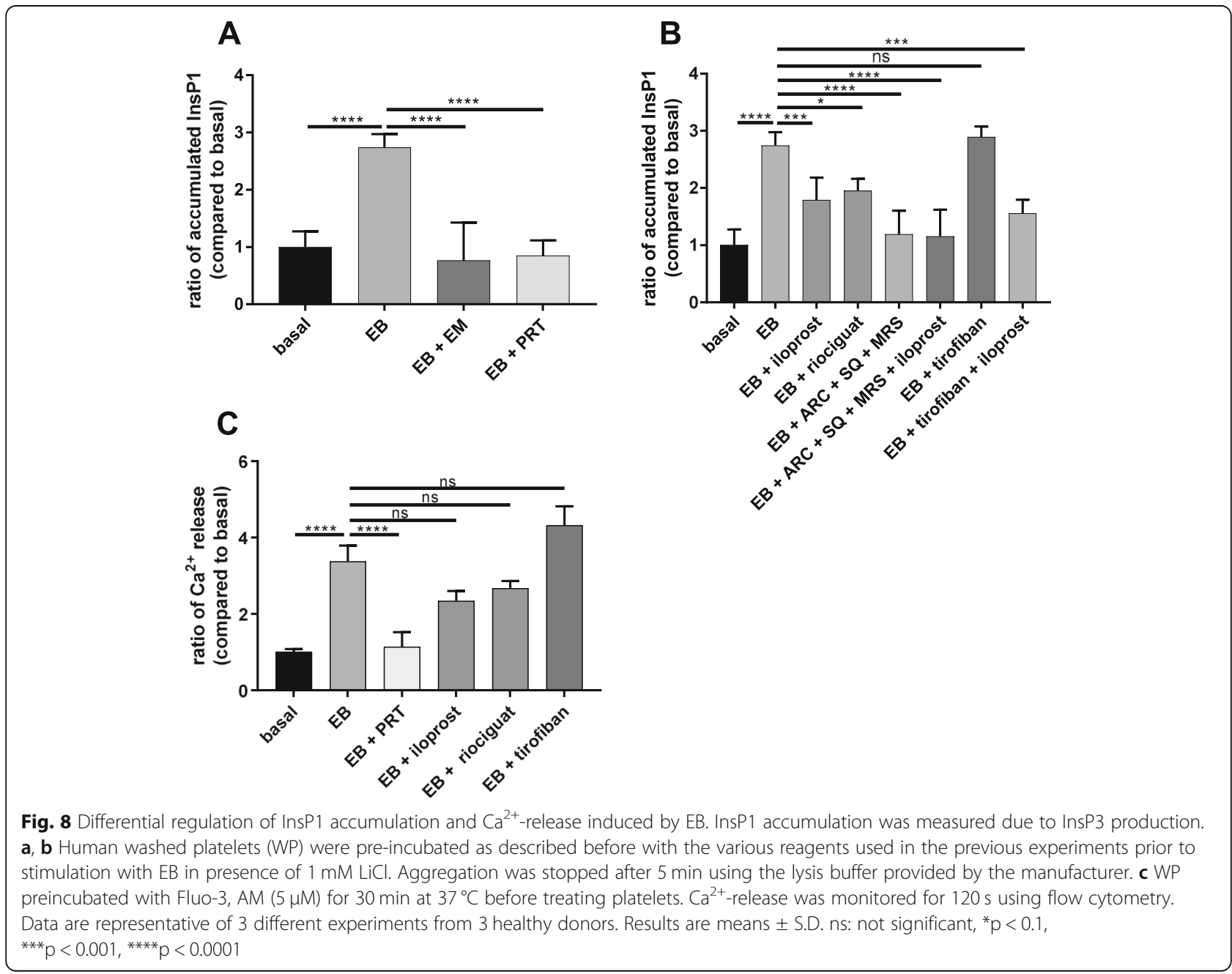


GPIb $\alpha$-mediated Syk stimulation and activation of human platelets. To ensure selective GPIb $\alpha$-activation the specific GPIb $\alpha$-ligand echicetin was used as multimer complex, coated on polystyrene beads (EB), leading to integrin $\alpha I I b \beta 3$-dependent aggregation of human platelets [39-41]. Echicetin monomer (EM) antagonized all observed EB effects but not GPVI/FcR $\gamma$ or GPCRmediated platelet aggregation.

EB induced platelet Syk phosphorylation and aggregation, which requires SFKs as these effects are prevented by the SFK inhibitors PP2 and dasatinib. Syk activation by $\mathrm{EB}$ in human platelets as assessed by Y352 and Y525/526 phosphorylation, was rapid but transient, indicating substantial dephosphorylation. Interestingly, dephosphorylation of murine Syk at Y346 ( Y352 in the human kinase) by the TULA-2 protein-tyrosine phosphatase suppressed its activation in murine platelets [56].

As reported by us recently, EB strongly activated murine platelets, expressing only FcR $\gamma$-chain but not FcR $\gamma$ IIA, which required GPIba [41]. Our previous quantitative proteomic studies with human platelets demonstrated expression of FCER1G (8170 copies), FCGR2A (990 copies) and Syk (4900 copies) [57]. Within a major, still ongoing phosphoproteomic study (to be published) we recently detected multiple tyrosinephosphorylated proteins in EB-stimulated human platelets including dually Y-phosphorylated FCER1G (Y56 and Y76, both 1.8 fold increased phosphorylation). Tyrosine phosphorylation of FCGR2A was not detected.

Altogether, the data indicate that EB activation of the human platelet GPIb-complex stimulates Syk phosphorylation via an SFK-dependent mechanism, which is antagonized by EM. SFK-dependent Syk activation involves direct Syk Y352 phosphorylation and may also involve the platelet ITAM protein FcR $\gamma$-chain.

To validate a functional role of Syk for GPIbosignaling in human platelets, we tested two different Syk inhibitors, OXSI-2 and PRT-318. These have been described as Syk and platelet function inhibitors in studies of convulxin/GPVI signaling [48-50]. PRT-318 also prevented heparin-induced thrombocytopenia (HIT) and thrombosis in a mouse model [49]. Another novel orally bioavailable Syk inhibitor (BI1002494) prevented arterial thrombosis and thromboinflammatory brain infarction in a mouse model to a similar extent as did, in another mouse model, platelet-specific Syk deficiency [58]. In humans, the Syk inhibitor fostamatinib was recently FDA-approved for the treatment of thrombocytopenia in adult patients with chronic immune thrombocytopenia (ITP) [59].

OXSI-2 $(2 \mu \mathrm{M})$ and PRT-318 $(1 \mu \mathrm{M})$ abolished EBinduced aggregation and strongly inhibited EB-induced Syk Y525/526, but not Syk Y352, phosphorylation. These inhibitors also prevented EB-induced phosphorylation of the direct Syk substrate (PLCY2 at Y759) and a further downstream effector, Akt S473. Syk Y525/526 phosphorylation, primarily due to autophosphorylation [23, 24], is expected to be blocked by Syk inhibitors. In contrast, Syk Y348/Y352 phosphorylation catalyzed by SFKs in intact cells initiates Syk activation [23], and is not blocked by Syk inhibitors but by the SFK inhibitor PP2, as observed here in our studies. Absent inhibition of platelet agonist-induced Syk Y348/Y352 phosphorylation, catalyzed by SFKs, has been used as one specificity criteria for Syk inhibitors $[48,49]$. For the overall regulation/activation of Syk, the sites Y348/Y352 are considered more important than the activation loop sites Y525/Y526 since mutations of latter sites did not reduce Syk kinase activity [23, 24]. This indicates that Syk tyrosine phosphorylation alone cannot be equated with Syk kinase activity. The strong inhibitory effects of Syk inhibitors on EBinduced Syk Y525/526, PLCY2 Y759 phosphorylation, and EB-induced aggregation, indicate that EB activate human platelets by a Syk-dependent process.

Based on the inhibitory effects of the Syk inhibitors, EB-induced Akt phosphorylation requires Syk and therefore appears to be downstream of Syk activity. However, Akt is certainly not an immediate Syk target/direct substrate but is most likely phosphorylated in response to additional adapter proteins affecting PI3K [10], which is confirmed by our results that the PI3K inhibitor wortmannin abolished EB-mediated Akt S473 phosphorylation without inhibition of Syk tyrosine phosphorylation.

A recent paper reported that Syk activity is dispensable for platelet GPIb-IX-V signaling induced by ristocetin/ vWF [37] by showing unaffected phosphorylation of Syk at Y352 and of Akt at S473 by the Syk inhibitor PRT318. Using the same Syk inhibitor we confirmed that ristocetin/vWF-induced Syk Y352 phosphorylation is not affected. However, we could show that PRT-318 inhibited phosphorylation of Syk at Y525/526, of the Syk substrate LAT at Y191 and of Akt at S473 in washed human platelets, indicating that Syk plays an important role in EB- as well in ristocetin/vWF-mediated GPIb $\alpha$ signaling of human platelets.

The receptors GPVI and CLEC-2 activate platelets by a Syk-dependent mechanism and require the release of secondary mediators $\left(\mathrm{ADP}, \mathrm{TxA}_{2}\right)$ for a full response, with certain differences [10]. Therefore, the role of the secondary mediators for the EB responses studied here was investigated. EB-induced platelet aggregation was abolished when the ADP receptors ( $\mathrm{P}_{2} \mathrm{Y}_{12}$ and $\left.\mathrm{P} 2 \mathrm{Y}_{1}\right)$ and the $\mathrm{TxA}_{2}$ receptor were blocked. In contrast, Syk phosphorylation was not at all (Y352) or only partially (Y525/Y526) reduced, whereas PLCY2 Y759 and Akt S473 phosphorylation was strongly reduced. We have no clear explanation for the partial inhibition of Syk 526/ 
526 phosphorylation by the secondary mediators except to speculate that this site may also be affected by pathways other than SFKs. This has also been proposed for PLC $\gamma 2$ and its phosphorylation [35]. In our platelet phosphoproteomic studies, ADP only stimulated Syk serine phosphorylation with no detectable effect on Syk tyrosine phosphorylation [53], which was confirmed in our ongoing experiments (Makhoul S et al., unpublished data). Overall, our data show that ADP and TxA2 have no major effect on EB-induced Syk phosphorylation but, in contrast, are required for further downstream effects including PLCY2 Y759/Akt S473 phosphorylation and aggregation.

In addition to ADP and $\mathrm{TxA}_{2}$, other pathways such as integrin $\alpha_{\mathrm{IIb}} \beta_{3}$ activation influence various steps of platelet activation including ITAM (FcyRIIA)-dependent Syk stimulation $[18,60]$. To evaluate the possible contribution of $\alpha_{\mathrm{IIb}} \beta_{3}$ for EB-induced signaling, we compared platelets from normal controls and $\alpha_{\mathrm{II}} \beta_{3}$-deficient platelets from a patient with Glanzmann thrombasthenia. There was no difference between normal and $\alpha_{\mathrm{II}} \beta_{3}$-deficient platelets with respect to EB-induced Syk activation as indicated by Y352 and Y525/Y526 phosphorylation. Similarly, the $\alpha_{\mathrm{II}} \beta_{3}$ inhibitor tirofiban did not affect EBinduced Syk tyrosine phosphorylation and phosphorylation of the Syk substrate PLCY2 at Y759. These data show that EB/GPIb-mediated phosphorylation and activation of Syk is integrin $\alpha_{\mathrm{II}} \beta_{3}$-independent.

Platelet functions are tightly regulated by a network of intracellular pathways consisting of protein kinases/protein phosphatases and their substrates $[6,8,61]$, but the regulation of specific GPIb-mediated signaling in human platelets by the PKA/PKG inhibitory pathways is unclear. PKA-stimulated GPIbß phosphorylation (S166, now S191) has been observed in multiple studies with human platelets [52, 62, 63]. When tested in the Chinese hamster ovary cell (CHO), PKA phosphorylation of GPIbß S166 correlated with reduced binding of vWF to the GPIb-IX complex [62] whereas other studies did not observe a functional effect of this phosphosite [63]. Clearly, PKA phosphorylation of GPIbß S166 (S191) and of other sites within the GPIb-IX complex need to be reinvestigated in future studies. In our present studies, we investigated primarily the regulation of GPIb $\alpha$-mediated signaling and showed that both iloprost (cAMP pathway) and riociguat (cGMP pathway) dose-dependently inhibited EB-induced aggregation of washed human platelets, with maximal inhibition at $5 \mathrm{nM}$ iloprost and $20 \mu \mathrm{M}$ riociguat, respectively. These clinically-used drugs and our conditions have been extensively used, and characterized to achieve strong, but selective activation of PKA (iloprost) and PKG (riociguat) in human platelets, also monitored by established substrates [8, 52-54]. Using different concentrations of iloprost $(1 \mathrm{nM}, 2 \mathrm{nM})$ and riociguat $(10 \mu \mathrm{M}, 20 \mu \mathrm{M})$, which inhibited significantly
EB-induced aggregation, they did not inhibit, but instead enhanced/prolonged EB-induced Syk activation observed as Syk Y352 and Y525/526 phosphorylation (hyperphosphorylation). Enhanced phosphorylation of the direct Syk substrates PLC 2 (Y759) and LAT (Y191) was also detected. In contrast, EB-induced Akt S473 phosphorylation was abolished. These results show that the PKA/ PKG pathways do not prevent EB-induced Syk activation (Y352/ Y525/526) and Syk activity (PLC 2/LAT), whereas further downstream effects (Akt phosphorylation, platelet aggregation) are strongly inhibited. The observation that cAMP and cGMP abolish GPIbamediated aggregation but not Syk activation under these conditions suggests that the inhibitory PKA/PKG effects occur downstream of Syk activation, not at the level of Syk activation/Syk activity.

Syk-mediated PLCY2 activation and InsP3 and 1,2-diacylglycerol (DAG) generation are essential components of the GPVI-pathway in human and murine platelet, and most GPVI/ITAM-effects are thought be mediated by Syk $[10,13,18]$, perhaps with some special exceptions such as generation of reactive oxygen species [64]. In contrast, specific GPIb $\alpha$-stimulation of the Syk/PLC $\gamma 2$ system with InsP3/DAG generation/intracellular $\mathrm{Ca}^{2+}$ release and its functional roles remained unclear until now $[20,30,35,65]$. Our data conclusively show that $\mathrm{EB}$, via GPIb $\alpha-/$ Syk activation, induced a marked increase of InsP1 as marker of InsP3 generation and $\mathrm{Ca}^{2+}$ release in human platelets, which was completely GPIb $\alpha$-dependent (response abolished by EM), Sykdependent (abolished by PRT-318) and integrin $\alpha_{\mathrm{II}} \beta_{3^{-}}$ independent (unaffected by tirofiban). However, iloprost (cAMP) and riociguat (cGMP) pre-treatment only partially inhibited EB-stimulated InsP1 increase and $\mathrm{Ca}^{2+}$ release. These only partial inhibitory effects differ from the known strong PKA/PKG-mediated inhibition of the InsP3/ $/ \mathrm{Ca}^{2+}$ response by platelet GPCR (ADP, thrombin, $\mathrm{TxA}_{2}$ ) activation, which occurs at several sites including GPCR signaling, PLC $\beta$, and the InsP3 receptor or its associated protein IRAG $[8,9,61,66,67]$. ADP-/ thrombin- / $\mathrm{TxA}_{2}$-stimulated $\mathrm{Ca}^{2+}$-release from intracellular stores in human platelets was essentially abolished by the PKA and PKG pathway [66, 67]. Our present data therefore suggest that cAMP/cGMP inhibit EB/GPIb $\alpha$ platelet activation partially at the level of the InsP3/Ca ${ }^{2+}$ response but perhaps stronger at sites downstream of InsP3 production and $\mathrm{Ca}^{2+}$ elevation. Interestingly, earlier studies using PLCy2-deficient murine platelets and $\mathrm{vWF} /$ botrocetin suggested that $\mathrm{PLC} Y 2$ is not required [35] or only moderately involved in GPIb-V-IX signaling [65] indicating that additional and/or compensating mechanisms exist, perhaps activation of other phospholipases. In our present studies, EB-induced aggregation, PLC 2 Y759 phosphorylation, Akt S473 phosphorylation 
as well as InsP1 accumulation/InsP3 generation and $\mathrm{Ca}^{2+}$-release were found to be dependent on the secondary mediators $\mathrm{ADP}$ and $\mathrm{TxA}_{2}$, different from the initial Syk phosphorylation/activation. It is very likely that important functional responses, such as $\mathrm{Ca}^{2+}$-release from intracellular stores, are heterogeneous and controlled by several pathways and proteins. A possible hotspot for the integration of various in-going Syk signals and outgoing responses is the membrane protein LAT, an important Syk substrate and component of ITAM/Syk signaling in immune cells and also in platelets $[10,68]$.
Interestingly, we observed similar effects of cAMP/ PKA and cGMP/PKG pathway stimulation on GPVImediated Syk phosphorylation. Whereas convulxinstimulated platelet aggregation was completely inhibited by iloprost (cAMP) and riociguat (cGMP), convulxininduced Syk phosphorylation (Y352 and Y525/526) was not inhibited, but instead clearly enhanced/prolonged (hyperphosphorylation) by iloprost or riociguat pretreatment. Convulxin-stimulated PLCY2 Y759 phosphorylation was also enhanced by the cAMP/cGMP pathways, whereas Akt S473 phosphorylation was inhibited. In line

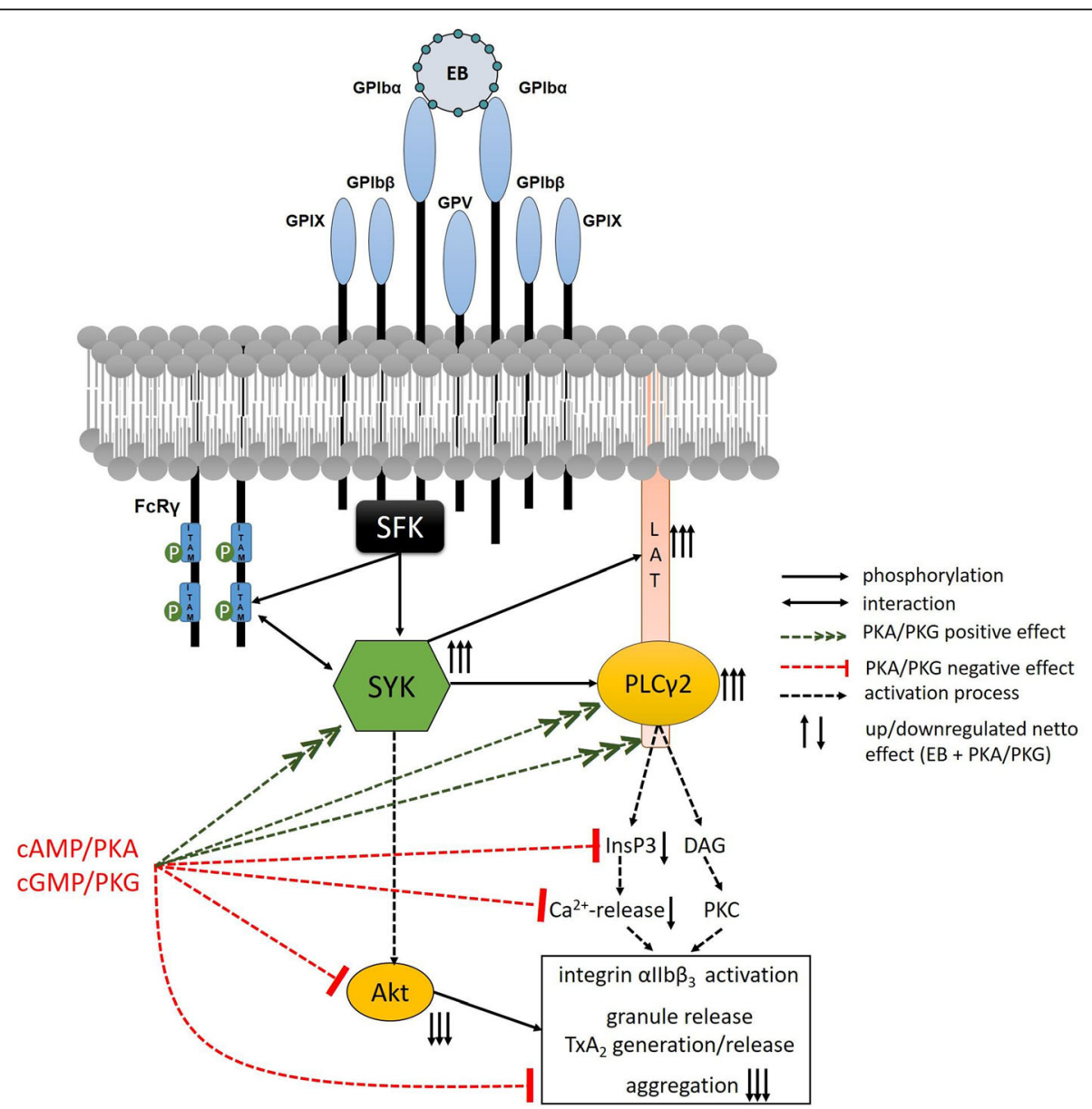

Fig. 9 CAMP/PKA and CGMP/PKG pathways cause dichotomous regulation of GPIba-mediated Syk stimulation and activation of human platelets. Selective binding of the snake venom toxin echicetin as multimeric complex (echicetin beads, EB) to the extracellular domain of GPIba leads to GPIba crosslinking and initiates a signaling cascade starting from activation of src family kinases (SFK). This GPIba activation results in tyrosine phosphorylation of ITAM-containing FCRy-chains and SFK-dependent phosphorylation and recruitment of the spleen tyrosine kinase (Syk), via its SH2 domains to tyrosine phosphorylated ITAMs produce full Syk activation. Syk-dependent phosphorylation and activation of the adaptor protein (LAT), phospholipase C Y2 and others lead to increased levels of InsP3 (IP3; measured here by its metabolite InsP1) and DAG, which are responsible for $\mathrm{Ca}^{2+}$-release and PKC activation (dotted black arrows). Additionally, Syk mediates indirectly the phosphorylation of Akt, one of Syk downstream effectors (dotted black arrow). Altogether, this leads to integrin activation, granule release and TxA2 synthesis and subsequent platelet aggregation. The major platelet inhibitory pathways represented by CAMP/PKA and CGMP/PKG strongly enhance EB-induced Syk phosphorylation/activation (dotted green arrows) and enhance Syk-mediated tyrosine phosphorylation of LAT and PLCY2 whereas InsP3 increase and Ca ${ }^{2+}$-release are partially inhibited, Akt phosphorylation is strongly inhibited (dotted red bars). The net effect of this crosstalk between platelet activation by EB and inhibition by cAMP/ PKA and CGMP/PKG is marked with up or down black arrows (arrow number reflects the intensity of the effect). Syk and its direct substrates PLCY2 and LAT are strongly activated (three arrows direction up), InsP3 and its subsequent $\mathrm{Ca}^{2+}$-release are partially inhibited (one arrow down). The phosphorylation of Akt is strongly inhibited by PKA and PKG-elevating agents, similar to the overall aggregation (three arrows direction down) 
with this observation, stimulation of CLEC-2 (a hemiITAM containing special adhesion receptor) on activated mouse platelets was shown to be only weakly inhibited by the cAMP, and not by the cGMP pathway [69]. Our cvx/ GPVI signaling data agree with the GPVI-signaling data of a recent published study, which reported that the cAMP/ PKA pathway inhibited distal but not proximal collagen/ GPVI-mediated signaling events in human platelets [70].

Presently, the mechanism(s) for the PKA/PKG-mediated increase of Syk Y352 and Y525/526 phosphorylation (hyperphosphorylation) and kinase activity is a topic of further investigation in our laboratory. Interestingly, a possibly related "Syk Y-hyperphosphorylation" has been observed in response to PKC inhibitors [71]. Recently, a large spectrum of additional Syk serine and tyrosine phosphorylation sites, interacting proteins and multisite ubiquitylation has been described [46, 72]. Clearly, additional regulatory mechanisms exist, which may control phosphorylation/dephosphorylation and membrane interactions of Syk, and which need to be addressed in future studies.

In contrast to Syk tyrosine phosphorylation, further downstream effects such as PI3K activation/Akt and aggregation in response to $\mathrm{EB}$ or convulxin were strongly inhibited by the PKA/PKG pathways or by blocking the secondary mediators ADP/TXA 2 . Both PKA/PKG pathways block platelet activation by $\mathrm{ADP}, \mathrm{TxA}_{2}$, thrombin $[8,9,67]$, which explains the inhibitory PKA/PKG effects

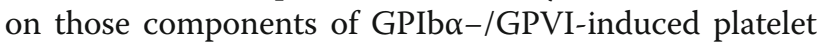
activation, which are dependent on secondary mediators. Previously it was suggested that several adhesion responses such as PI3K-dependent Akt activation are regulated by ADP-dependent and ADP-independent mechanisms [10, 73]. PKA and PKG are expected to inhibit ADP-dependent, but not ADP-independent PI3K/ Akt activation. Finally, it has been increasingly recognized that crucial platelet functions are controlled and inhibited at multiple checkpoints including several PKA and PKG checkpoints $[8,9,61]$. A model of our current understanding of the effect of cAMP/PKA and cGMP/ PKG on the GPIb $\alpha$-regulated Syk network in human platelets is shown in Fig. 9.

\section{Conclusion}

Our data establish that selective activation of GPIb $\alpha$ in human platelets by using echicetin-beads results in SFKdependent Syk activation, subsequent Syk-mediated signaling, and ultimately integrin-dependent aggregation. In contrast to others [37] we obtained evidence that Syk plays an important role in EB- as well in ristocetin/vWFmediated GPIb $\alpha$-signaling of human platelets.

Syk pathway components display a variable dependency on secondary mediators (ADP, TxA2) and variable regulation by cAMP/cGMP inhibitory pathways, revealing two distinct directions of GPIb $\alpha-/$ Syk signaling. The initial GPIb $\alpha$-caused Syk phosphorylation, activation and direct substrate phosphorylation is independent of the integrin $\alpha \operatorname{IIb} \beta 3$ and secondary mediators. Then, two distinct platelet inhibitory mediators, cAMP/PKA and cGMP/PKG,

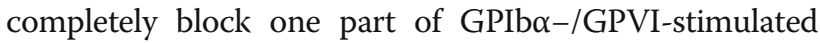
Syk signaling responses (aggregation, PI3K pathway), but increase the initial receptor-induced Syk activation and phosphorylation of two important direct Syk substrates, PLCY 2 and LAT. The observation that cAMP and cGMP abolish GPIb $\alpha$-mediated aggregation but not Syk activation under our conditions suggests that the inhibitory PKA/PKG effects occur downstream of Syk activation, not at the level of Syk activation/Syk activity. Our results indicate the presence of distinct Syk signaling/effector systems, which differ in their interaction with other signaling pathways. The physiological and pathophysiological significance of these different Syk effector systems needs further investigation. This is clearly warranted in the light of the functional importance of Syk in platelets and immune cells, and in the light of Syk and related tyrosine protein kinases as important pharmacological target.

\section{Additional files}

\begin{abstract}
Additional file 1: Figure S1. Echicetin purification and qualitative validation. Echicetin was isolated from the snake venom of Echis carinatus sochureki by affinity chromatography followed by anion exchange chromatography using DEAE column. a Elution was performed with a gradient of $\mathrm{NaCl}$, from 0 to $1 \mathrm{M}$ at a flow rate of $1 \mathrm{ml} / \mathrm{min}$. Two main peaks were separately eluted (P1 and P2) at around 62.5 and $167 \mathrm{mM}$, respectively. $\mathbf{b}$ Samples from $\mathrm{P} 1$ and $\mathrm{P} 2$ were analyzed by silver staining under non-reducing (NR) and reducing conditions (R) in a 15\% SDS-gel. Bands detected under reducing conditions were cut from the gel, digested using trypsin, and analyzed by MS-analysis. The upper and lower bands of peak 1 were identified under the Uniprot IDs: P81017 (echicetin a-subunit) and P81996 (echicetin $\beta$-subunit), respectively. However, the upper band of peak 2 was detected as a sequence not related to Echis carinatus sochureki species, and the lower band was identified under the Uniprot ID: P81996 (echicetin $\beta$-subunit). For all experiments, echicetin from peak 1 was used. c Representative aggregation curves of human washed platelets (WP), which were stimulated under stirring conditions with $\mathrm{EB}$ or BSA-coated beads (as negative control). WP were preincubated with echicetin (EM) $(25 \mu \mathrm{g} / \mathrm{ml} ; 3 \mathrm{~min})$ or with tirofiban $(1.25 \mu \mathrm{g}$ / $\mathrm{ml} ; 1 \mathrm{~min}$ ) prior to stimulation with EB. $\mathbf{d}$ Corresponding quantitative data of platelet aggregation expressed as maximum percentage of light transmission. Results are shown as means \pm S.D. of 3 independent experiments with platelets from 3 healthy donors (**** $p<0.0001$ ). (PPTX $520 \mathrm{~kb}$ )
\end{abstract}

Additional file 2: Figure S2. Echicetin monomer does not affect aggregation induced by GPVI or by G-protein coupled receptors. Washed platelets were pre-incubated with $25 \mu \mathrm{g} / \mathrm{ml}$ echicetin monomer for $3 \mathrm{~min}$ before stimulating with a collagen $(3 \mu \mathrm{g} / \mathrm{ml})$, c ADP $(1.75 \mu \mathrm{M})$, e TxA2 (60 $\mathrm{nM}), \mathbf{g}$ TRAP6 $(5 \mu \mathrm{M})$ and $\mathbf{i}-\mathbf{k}$ thrombin $(0.1$ or $0.03 \mathrm{U} / \mathrm{ml})$. Quantitative data are presented as means \pm SD for $\mathbf{b}$ collagen, $\mathbf{d}$ ADP, $\mathbf{f}$ TXA2, $\mathbf{h}$ TRAP- $6, \mathbf{j}, \mathbf{l}$ thrombin. Data are presented from 3 different experiments with platelets from 3 different healthy donors; ns: not significant. (PPTX $174 \mathrm{~kb}$ )

Additional file 3: Figure S3. EB-induced platelet aggregation is dependent on src family kinases (SFKs). Washed human platelets (WP) were pre-incubated for 5 min with the SFK inhibitor, PP2 $(10 \mu \mathrm{M})$ or with its inactive analogue, PP3 $(10 \mu \mathrm{M})$ prior to stimulation with EB. a Representative curves of the effect of PP2 and PP3 on platelet 
aggregation and $\mathbf{b}$ the corresponding quantification are shown as means \pm S.D. Data are from at least 3 independent experiments with platelets from at least 3 healthy donors; ns: not significant, ${ }^{* * *} p<0.0001$. (PPTX $70 \mathrm{~kb}$ )

Additional file 4: Figure S4. Syk inhibitors diminish EB-induced general tyrosine phosphorylation and VWF/ristocetin-induced platelet aggregation and phosphorylation of Syk 525/526, LAT Y191 and Akt S473. a Washed human platelets (WP) were pre-incubated with 2 different Syk inhibitors, OXSI-2 $(2 \mu \mathrm{M})$ and PRT-318 $(1 \mu \mathrm{M})$ for 5 min prior to stimulation with EB. Aggregation was stopped after 1,2 or 5 min using Laemmli buffer. General tyrosine phosphorylation was analyzed by western blot using a pan-phosphotyrosine antibody. b WP were pre-incubated for 5 min with vehicle control or PRT-318 $(1 \mu \mathrm{M})$ in the presence or absence of $1.25 \mu \mathrm{g} /$ $\mathrm{ml}$ tirofiban prior to stimulation with $2.5 \mu \mathrm{g} / \mathrm{ml}$ human vWF plus $0.5 \mathrm{mg} /$ $\mathrm{ml}$ ristocetin. Representative curves show the effect of PRT-318 on vWF/ R-induced platelet aggregation. Incubation of WP with tirofiban was used to dissect between vWF-mediated platelet agglutination and integrin $a_{\| b} \beta_{3}$-dependent aggregation. $\mathbf{c}$ The corresponding quantification demonstrates the effect of PRT-318 on the residual platelet aggregation response (overall maximum light transmission in the absence of tirofiban minus maximum light transmission in the presence of tirofiban) induced by vWF/risotcetin. Results are calculated from 4 different experiments with platelets from 4 healthy volunteers and data are presented as means \pm S.D. ${ }^{* *} p<0.01,{ }^{* * *} p<0.001,{ }^{* * * *} p<0.0001$. d, e Platelet aggregation was stopped after $2 \mathrm{~min}$ by addition of Laemmli buffer to analyze Syk Y525/526, Y352 and LAT Y 191 and aggregation was stopped after 5 min for the analysis of Akt $\$ 473$ by immunoblotting. Western blots are representative for at least 3 independent experiments from at least 3 healthy volunteers. (PPTX $380 \mathrm{~kb}$ )

Additional file 5: Figure S5. Iloprost and riociguat inhibit EB-induced platelet aggregation in a dose-dependent manner and induce stable VASP S157 and S239 phosphorylation. Representative curves of the effect of increased concentrations of $\mathbf{a}$ iloprost and $\mathbf{b}$ riociguat on EB-induced platelet aggregation. $\mathbf{c}$ Washed human platelets (WP) were pre-incubated for 3 and $2 \mathrm{~min}$ with $2 \mathrm{nM}$ iloprost and $20 \mu \mathrm{M}$ riociguat, respectively prior to stimulation with EB. VASP phosphorylation at S239 (the PKG preferred, but also PKA site) was analyzed by immunoblotting. VASP S157 phosphorylation at S157 (the PKA preferred, but also PKG site) is visible here by the well-established pS157-dependent shift from the $46 \mathrm{kDa}$ to $50 \mathrm{kDa}$ form of VASP in SDS-PAGE. The complete shift of the VASP to the $50 \mathrm{kDa}$ form indicates near stoichiometric VASP S157 phosphorylation by iloprost-activated PKA. Quantification VASP S239 is represented as ratio compared to the loading control $\beta$-actin. Data are presented of 3 different experiments with platelets from 3 healthy volunteers as means \pm S.D. ${ }^{* * *} p<0.0001$. (PPTX $292 \mathrm{~kb}$ )

Additional file 6: Figure S6. Syk and PLCY2 tyrosine phosphorylation is also increased and prolonged at $1 \mathrm{nM}$ iloprost. Washed human platelets (WP) were pre-incubated $3 \mathrm{~min}$ at $37^{\circ} \mathrm{C}$ in the presence or absence of iloprost ( $1 \mathrm{nM}$ ) prior to stimulation with EB. Phosphorylation of a Syk Y525/526 and Y352, d PLCY2 Y759 and Akt S473 was analyzed by immunoblotting in a time dependent manner. $\mathbf{b}, \mathbf{c}, \mathbf{e}, \mathbf{f}$ Quantification of the phosphorylated proteins are represented as ratio compared to the corresponding total protein. Data are shown of 3 different experiments with platelets from 3 healthy volunteers as means \pm S.D. ns: not significant, ${ }^{*} p<0.05,{ }^{* *} p<0.01,{ }^{* * * *} p<0.0001$. (PPTX $1770 \mathrm{~kb}$ )

Additional file 7: Figure S7. Syk and PLCY2 tyrosine phosphorylation is also increased and prolonged at $10 \mu \mathrm{M}$ riociguat. Washed human platelets (WP) were pre-incubated for $5 \mathrm{~min}$ at $37^{\circ} \mathrm{C}$ with vehicle control (DMSO) and $10 \mu \mathrm{M}$ riociguat, respectively prior to stimulation with $\mathrm{EB}$. Phosphorylation of a Syk Y525/526 and Y352, d PLCY2 Y759 and Akt S473 were analyzed by immunoblotting in a time dependent manner. b c c, e, f Quantification of the phosphorylated proteins are represented as ratio compared to the corresponding total protein. Data are shown of 3 different experiments with platelets from 3 healthy volunteers as means \pm S.D. ns: not significant, ${ }^{*} \mathrm{p}<0.05,{ }^{* *} \mathrm{p}<0.01,{ }^{* * *} p<0.001,{ }^{* * * *} \mathrm{p}<0.0001$.

(Samples were loaded on the same gel; a black line was used to indicate that a group of samples not related to this data set was not shown). (PPTX $358 \mathrm{~kb}$ )

Additional file 8: Figure S8. lloprost and riociguat inhibit convulxininduced platelet aggregation but not Syk activation. Washed human platelets (WP) were pre-incubated with iloprost ( $2 \mathrm{nM}, 3 \mathrm{~min}$ ) or riociguat ( $20 \mu \mathrm{M}, 2 \mathrm{~min}$ ) prior to stimulation with $50 \mathrm{ng} / \mathrm{ml}$ convulxin. a Representative aggregation curves and the corresponding quantitative data are shown in b. c Aggregation was stopped after 1,2 or 5 min using Laemmli buffer. Syk Y525/526 and Y352 were analyzed by western blot d, e Quantification of the phosphorylated proteins is presented as ratio compared to the total Syk protein. Results are representative of at least 3 different experiments with platelets from at least 3 healthy volunteers, data are presented as means \pm S.D. ns: not significant, ${ }^{* * * *} p<0.0001$. (PPTX $181 \mathrm{~kb})$

\section{Abbreviations}

ADP: Adenosine diphosphate; Akt: Protein kinase B; cAMP: cyclic Adenosine Monophosphate; CGMP: cyclic Guanosine Monophosphate; CLEC-2: C-type lectin-2; EB: Echicetin Beads; EM: Echicetin Monomers; GPCRs: G proteincoupled receptors; GPIba: Glycoprotein Iba; InsP1/3: Inositol monophosphate/ triphosphate; ITAM: Immunoreceptor tyrosine-based activation motif; kDa: kilo Dalton; LAT: Linker of Activated T cells; NO: Nitric Oxide; PGI 2 : Prostaglandin I2; PKA: Protein Kinase A; PKG: Protein Kinase Gi PLCY: Phospholipase CY; SFK: Src- Family Kinase; Src: Sarcoma; Syk: Spleen tyrosine kinase; TXA

\section{Acknowledgements}

SG was supported by a grant from Ministry of Science and Higher Education of the Russian Federation (№AAAA-A18-118012290371-3). The authors thank Dr. A. Navdaev (CSL Behring AG, Zurich) for his help and advice in preparing echicetin proteins and Dr. Suzanne M. Lohmann for English editing.

\section{Authors' contributions}

SM performed most of the experiments, analyzed and interpreted data and wrote the manuscript (part of her doctoral thesis). KT performed, analyzed and interpreted initial experiments (part of her doctoral thesis). SG designed experiments, interpreted data and revised the manuscript. ST was responsible for mass spectrometry experiments and data. DP recruited patients. UW designed the study, interpreted data and wrote the manuscript. $\mathrm{KJ}$ designed the study and experiments, interpreted data, wrote and revised the manuscript. All authors read and approved the final manuscript.

\section{Funding}

The study was supported by the German Federal Ministry of Education and Research (BMBF $01 \mathrm{EO} 1003$ and $01 \mathrm{EO} 1503$ (K, UW)).

\section{Availability of data and materials}

Datasets and non-commercial materials can be obtained from the corresponding author on reasonable request.

\section{Ethics approval and consent to participate}

All studies using human platelets from healthy volunteers and from a patient with Glanzmann thrombasthenia caused by a homozygous point mutation in ITGA2B c.621C > T; p.T176I [42, 43] were approved by the local ethics committees (Study No. 837.302.12; 25.07.12; FF109/2015).

\section{Consent for publication}

Not applicable.

\section{Competing interests}

The authors declare that they have no competing interests.

\section{Author details}

${ }^{1}$ Center for Thrombosis and Hemostasis (CTH), University Medical Center Mainz of the Johannes Gutenberg University Mainz, Mainz, Germany. ${ }^{2}$ Sechenov Institute of Evolutionary Physiology and Biochemistry, Russian Academy of Sciences, St. Petersburg, Russia. ${ }^{3}$ Core Facility for Mass Spectrometry, Institute for Immunology, University Medical Center Mainz, Mainz, Germany. ${ }^{4}$ DKD HELIOS Klinik Wiesbaden GmbH, Wiesbaden, Germany. 


\section{Received: 2 May 2019 Accepted: 29 August 2019}

\section{Published online: 13 September 2019}

\section{References}

1. Versteeg HH, Heemskerk JWM, Levi M, Reitsma PH. NEW FUNDAMENTALS IN HEMOSTASIS. Physiol Rev. 2013;93(1):327-58.

2. Franco AT, Corken A, Ware J. Platelets at the interface of thrombosis, inflammation, and cancer. Blood. 2015;126(5):582-8.

3. Jurk K. Analysis of platelet function and dysfunction. Hamostaseologie. 2015; 35(1):60-72.

4. Offermanns S. Activation of platelet function through G protein-coupled receptors. Circ Res. 2006;99(12):1293-304.

5. Senis YA, Mazharian A, Mori J. Src family kinases: at the forefront of platelet activation. Blood. 2014;124(13):2013-24.

6. Brass LF, Ma P, Tomaiuolo M, Diamond SL, Stalker TJ. A Systems Approach to the Platelet Signaling Network and the Hemostatic Response to Injury. Platelets in Thrombotic and Non-Thrombotic Disorders: Springer. 2017:36778.

7. Smolenski A. Novel roles of CAMP/CGMP-dependent signaling in platelets. J Thromb Haemost. 2012;10(2):167-76.

8. Makhoul S, Walter E, Pagel O, Walter U, Sickmann A, Gambaryan S, et al. Effects of the NO/soluble guanylate cyclase/cGMP system on the functions of human platelets. Nitric oxide : biology and chemistry. 2018;76:71-80.

9. Nagy Z, Smolenski A. Cyclic nucleotide-dependent inhibitory signaling interweaves with activating pathways to determine platelet responses. Research and Practice in Thrombosis and Haemostasis. 2018:2(3):558-71.

10. Moroi AJ, Watson SP. Impact of the PI3-kinase/Akt pathway on ITAM and hemITAM receptors: Haemostasis, platelet activation and antithrombotic therapy. Biochem Pharmacol. 2015;94(3):186-94.

11. Poole A, Gibbins JM, Turner M, van Vugt MJ, van de Winkel JG, Saito T, et al. The Fc receptor gamma-chain and the tyrosine kinase Syk are essential for activation of mouse platelets by collagen. EMBO J. 1997;16(9):2333-41.

12. Nieswandt B, Bergmeier W, Schulte V, Rackebrandt K, Gessner JE, Zirngibl H. Expression and function of the mouse collagen receptor glycoprotein $\mathrm{VI}$ is strictly dependent on its association with the FCR gamma chain. J Biol Chem. 2000;275(31):23998-4002.

13. Rayes J, Watson SP, Nieswandt B. Functional significance of the platelet immune receptors GPVI and CLEC-2. J Clin Invest. 2019;129(1):12-23.

14. Gardiner EE, Andrews RK. Platelet adhesion. In: Gresele P, Kleiman NS, Lopez $J A$, Page CP, editors. Platelets in thrombotic and non-thrombotic disorders: pathophysiology, pharmacology and therapeutics: an update. Cham: Springer International Publishing; 2017. p. 309-19.

15. Ozaki Y, Suzuki-Inoue $K$, Inoue O. Platelet receptors activated via mulitmerization: glycoprotein VI, GPIb-IX-V, and CLEC-2. J Thromb Haemost. 2013;11(Suppl 1):330-9.

16. Kurosaki T, Johnson SA, Pao L, Sada K, Yamamura H, Cambier JC. Role of the SYK AUTOPHOSPHORYLATION site and SH2 domains in b-cell antigen receptor signaling. J Exp Med. 1995;182(6):1815-23.

17. Johnson SA, Pleiman CM, Pao L, Schneringer J, Hippen K, Cambier JC. Phosphorylated IMMUNORECEPTOR signaling motifs (ITAMS) exhibit unique abilities to bind and activate LYN and SYK tyrosine kinases. J Immunol. 1995 155(10):4596-603.

18. Mocsai A, Ruland J, Tybulewicz VLJ. The SYK tyrosine kinase: a crucial player in diverse biological functions. Nat Rev Immunol. 2010;10(6):387-402.

19. Falati S, Edmead CE, Poole AW. Glycoprotein Ib-V-IX, a receptor for von Willebrand factor, couples physically and functionally to the fc receptor gamma-chain, Fyn, and Lyn to activate human platelets. Blood. 1999;94(5): 1648-56.

20. López JA. The platelet glycoprotein Ib-IX-V complex. In: Gresele P, Kleiman NS, Lopez JA, Page CP, editors. Platelets in thrombotic and non-thrombotic disorders: pathophysiology, pharmacology and therapeutics: an update. Cham: Springer International Publishing; 2017. p. 85-97.

21. Stegner D, Haining EJ, Nieswandt B. Targeting glycoprotein VI and the Immunoreceptor tyrosine-based activation motif signaling pathway. Arteriosclerosis Thrombosis and Vascular Biology. 2014:34(8):1615-20.

22. Zeiler M, Moser M, Mann M. Copy number analysis of the murine platelet proteome spanning the complete abundance range. Mol Cell Proteomics. 2014;13(12):3435-45.

23. Bradshaw JM. The Src, Syk, and Tec family kinases: distinct types of molecular switches. Cell Signal. 2010;22(8):1175-84.
24. Tsang E, Giannetti AM, Shaw D, Dinh M, Tse JKY, Gandhi S, et al. Molecular mechanism of the Syk activation switch. J Biol Chem. 2008:283(47):32650-9.

25. Gradler U, Schwarz D, Dresing V, Musil D, Bomke J, Frech M, et al. Structural and biophysical characterization of the Syk activation switch. J Mol Biol. 2013;425(2):309-33.

26. Kurosaki T, Hikida M. Tyrosine kinases and their substrates in B lymphocytes. Immunol Rev. 2009;228:132-48.

27. Geahlen RL. Getting Syk: spleen tyrosine kinase as a therapeutic target. Trends Pharmacol Sci. 2014;35(8):414-22.

28. Larive RM, Urbach S, Poncet J, Jouin P, Mascre G, Sahuquet A, et al. Phosphoproteomic analysis of Syk kinase signaling in human cancer cells reveals its role in cell-cell adhesion. Oncogene. 2009;28(24):2337-47.

29. Naldi A, Larive RM, Czerwinska U, Urbach S, Montcourrier P, Roy C, et al. Reconstruction and signal propagation analysis of the Syk signaling network in breast cancer cells. Plos Computational Biology. 2017;13(3).

30. Kasirer-Friede A, Cozzi MR, Mazzucato M, De Marco L, Ruggeri ZM, Shattil SJ. Signaling through GP Ib-IX-V activates alpha IIb beta 3 independently of other receptors. Blood. 2004;103(9):3403-11.

31. Bergmeier W, Chauhan AK, Wagner DD. Glycoprotein Ib alpha and von Willebrand factor in primary platelet adhesion and thrombus formation: lessons from mutant mice. Thromb Haemost. 2008:99(2):264-70.

32. Ozaki Y, Asazuma N, Suzuki-Inoue K, Berndt MC. Platelet GPIb-IX-Vdependent signaling. J Thromb Haemost. 2005;3(8):1745-51.

33. Marshall SJ, Senis YA, Auger JM, Feil R, Hofmann F, Salmon G, et al. GPIbdependent platelet activation is dependent on Src kinases but not MAP kinase or cGMP-dependent kinase. Blood. 2004;103(7):2601-9.

34. Gardiner EE, Arthur JF, Shen Y, Karunakaran D, Moore LA, Esch JSA, et al. GPIb alpha-selective activation of platelets induces platelet signaling events comparable to GPVI activation events. Platelets. 2010;21(4):244-52.

35. Suzuki-Inoue K, Wilde Jl, Andrews RK, Auger JM, Siraganian RP, Sekiya F, et al. Glycoproteins VI and Ib-IX-V stimulate tyrosine phosphorylation of tyrosine kinase Syk and phospholipase Cgamma2 at distinct sites. The Biochemical journal. 2004;378(Pt 3):1023-9.

36. Li ZY, Xi XD, Gu MY, Feil R, Ye RD, Eigenthaler M, et al. A stimulatory role for cGMP-dependent protein kinase in platelet activation. Cell. 2003;112(1):7786.

37. Badolia R, Kostyak JC, Dangelmaier C, Kunapuli SP. Syk Activity Is Dispensable for Platelet GP1b-IX-V Signaling. International journal of molecular sciences. 2017;18(6).

38. Gibbins JM. Platelet adhesion signalling and the regulation of thrombus formation. J Cell Sci. 2004;117(16):3415-25.

39. Navdaev A, Subramanian H, Petunin A, Clemetson KJ, Gambaryan S, Walter U. Echicetin coated polystyrene beads: a novel tool to investigate GPIbspecific platelet activation and aggregation. PLoS One. 2014;9(4):e93569.

40. Navdaev A, Dormann D, Clemetson JM, Clemetson KJ. Echicetin, a GPIbbinding snake C-type lectin from Echis carinatus, also contains a binding site for lgM kappa responsible for platelet agglutination in plasma and inducing signal transduction. Blood. 2001;97(8):2333-41.

41. Trabold K, Makhoul S, Gambaryan S, van Ryn J, Walter U, Jurk K. The direct thrombin inhibitors dabigatran and Lepirudin inhibit GPIbalpha-mediated platelet aggregation. Thromb Haemost. 2019;119(6):916-29.

42. Geffen JPV, Brouns SLN, Batista J, McKinney H, Kempster C. Nagy M, et al. Haematologica: High-throughput elucidation of thrombus formation reveals sources of platelet function variability; 2018.

43. Westrup D, Santoso S, Follert-Hagendorff K, Bassus S, Just M, Jablonka B, et al. Glanzmann thrombasthenia Frankfurt I is associated with a point mutation Thr176lle in the N-terminal region of alpha Ilb subunit integrin. Thromb Haemost. 2004;92(5):1040-51.

44. Estevez B, Kim K, Delaney MK, Stojanovic-Terpo A, Shen B, Ruan CG, et al. Signaling-mediated cooperativity between glycoprotein Ib-IX and proteaseactivated receptors in thrombin-induced platelet activation. Blood. 2016; 127(5):626-36

45. Zhang J, Billingsley ML, Kincaid RL, Siraganian RP. Phosphorylation of Syk activation loop tyrosines is essential for Syk function - an in vivo study using a specific anti-Syk activation loop phosphotyrosine antibody. J Biol Chem. 2000:275(45):35442-7.

46. Bohnenberger $\mathrm{H}$, Oellerich $\mathrm{T}$, Engelke $\mathrm{M}, \mathrm{Hsiao} H \mathrm{H}$, Urlaub H, Wienands J. Complex phosphorylation dynamics control the composition of the Syk interactome in B cells. Eur J Immunol. 2011;41(6):1550-62.

47. Gnoni A, Marech I, Silvestris N, Vacca A, Lorusso V. Dasatinib: an anti-tumour agent via Src inhibition. Curr Drug Targets. 2011;12(4):563-78. 
48. Bhavaraju K, Kim S, Daniel JL, Kunapuli SP. Evaluation of 3-(1-methyl-1Hindol-3-yl-methylene)-2-oxo-2, 3-dihydro-1H-indole-5-sulfonamide (OXSI-2), as a Syk-selective inhibitor in platelets. Eur J Pharmacol. 2008;580(3):285-90.

49. Reilly MP, Sinha U, Andre P, Taylor SM, Pak Y, DeGuzman FR, et al. PRT060318, a novel Syk inhibitor, prevents heparin-induced thrombocytopenia and thrombosis in a transgenic mouse model. Blood. 2011;117(7):2241-6.

50. Andre P, Morooka T, Sim D, Abe K, Lowell C, Nanda N, et al. Critical role for Syk in responses to vascular injury. Blood. 2011;118(18):5000-10.

51. Loroch S, Trabold K, Gambaryan S, Reiss C, Schwierczek K, Fleming I, et al. Alterations of the platelet proteome in type I Glanzmann thrombasthenia caused by different homozygous delG frameshift mutations in ITGA2B. Thromb Haemost. 2017;117(3):556-69.

52. Beck F, Geiger J, Gambaryan S, Veit J, Vaudel M, Nollau P, et al. Timeresolved characterization of CAMP/PKA-dependent signaling reveals that platelet inhibition is a concerted process involving multiple signaling pathways. Blood. 2014;123(5):e1-e10.

53. Beck F, Geiger J, Gambaryan S, Solari FA, Dell'Aica M, Loroch S, et al. Temporal quantitative phosphoproteomics of ADP stimulation reveals novel central nodes in platelet activation and inhibition. Blood. 2017:129(2):E1E12.

54. Reiss C, Mindukshev I, Bischoff V, Subramanian H, Kehrer L, Friebe A, et al. The SGC stimulator riociguat inhibits platelet function in washed platelets but not in whole blood. Br J Pharmacol. 2015;172(21):5199-210.

55. Gilio K, Munnix ICA, Mangin P, Cosemans J, Feijge MAH, Van der Meijden PEJ, et al. Non-redundant roles of phosphoinositide 3-kinase isoforms alpha and beta in glycoprotein Vl-induced platelet signaling and Thrombus formation. J Biol Chem. 2009;284(49):33750-62.

56. Reppschlager K, Gosselin J, Dangelmaier CA, Thomas DH, Carpino N McKenzie SE, et al. TULA-2 protein phosphatase suppresses activation of Syk through the GPVI platelet receptor for collagen by dephosphorylating Tyr(P)(346), a regulatory site of Syk. J Biol Chem. 2016;291(43):22427-41.

57. Burkhart JM, Vaudel M, Gambaryan S, Radau S, Walter U, Martens L, et al. The first comprehensive and quantitative analysis of human platelet protein composition allows the comparative analysis of structural and functional pathways. Blood. 2012;120(15):e73-82.

58. van Eeuwijk JMM, Stegner D, Lamb DJ, Kraft P, Beck S, Thielmann I, et al. The Novel Oral Syk Inhibitor, Bl1002494, Protects Mice From Arterial Thrombosis and Thromboinflammatory Brain Infarction. Arteriosclerosis Thrombosis and Vascular Biology. 2016;36(6):1247-+.

59. Fueyo J, Alonso MM, Kerrigan BCP, Gomez-Manzano C. Linking inflammation and cancer: the unexpected SYK world. Neuro-Oncology. 2018;20(5):582-3.

60. Boylan B, Gao C, Rathore V, Gill JC, Newman DK, Newman PJ. Identification of fc gamma Rlla as the ITAM-bearing receptor mediating alpha Ilb beta 3 outside-in integrin signaling in human platelets. Blood. 2008;112(7):2780-6.

61. Stefanini $L$, Bergmeier $W$. Negative regulators of platelet activation and adhesion. J Thromb Haemost. 2018;16(2):220-30.

62. Bodnar RJ, Xi XD, Li ZY, Berndt MC, Du XP. Regulation of glycoprotein Ib-IXvon Willebrand factor interaction by cAMP-dependent protein kinasemediated phosphorylation at Ser(166) of glycoprotein Ib beta. J Biol Chem. 2002;277(49):47080-7.

63. David T, Strassel C, Eckly A, Cazenave JP, Gachet C, Lanza F. The platelet glycoprotein GPIb beta intracellular domain participates in von Willebrand factor induced-filopodia formation independently of the Ser 166 phosphorylation site. J Thromb Haemost. 2010;8(5):1077-87.

64. Arthur JF, Qiao J, Shen Y, Davis AK, Dunne E, Berndt MC, et al. ITAM receptor-mediated generation of reactive oxygen species in human platelets occurs via Syk-dependent and Syk-independent pathways. J Thromb Haemost. 2012;10(6):1133-41.

65. Mangin P, Yuan Y, Goncalves I, Eckly A, Freund M, Cazenave JP, et al. Signaling role for phospholipase C gamma 2 in platelet glycoprotein $\mathrm{lb}$ alpha calcium flux and cytoskeletal reorganization. Involvement of a pathway distinct from FcR gamma chain and fc gamma RIIA.J Biol Chem. 2003:278(35):32880-91.

66. Geiger J, Nolte C, Butt E, Sage SO, Walter U. Role Of Cgmp And CgmpDependent Protein-Kinase In Nitrovasodilator Inhibition Of Agonist-Evoked Calcium Elevation In Human Platelets. Proc Natl Acad Sci U S A. 1992;89(3): 1031-5

67. Geiger J, Nolte C, Walter U. Regulation Of Calcium Mobilization And Entry In Human Platelets By Endothelium-Derived Factors. Am J Physiol. 1994;267(1): C236-CC44.
68. Judd BA, Myung PS, Obergfell A, Myers EE, Cheng AM, Watson SP, et al. Differential requirement for LAT and SLP-76 in GPVI versus T cell receptor signaling. J Exp Med. 2002;195(6):705-17.

69. Borgognone A, Navarro-Nunez L, Correia JN, Pollitt AY, Thomas SG, Eble JA, et al. CLEC-2-dependent activation of mouse platelets is weakly inhibited by cAMP but not by cGMP. J Thromb Haemost. 2014;12(4):550-9.

70. Clark JC, Kavanagh DM, Watson S, Pike JA, Andrews RK, Gardiner EE, et al. Adenosine and Forskolin inhibit platelet aggregation by collagen but not the proximal Signalling events. Thromb Haemost. 2019;119(7):1124-37.

71. Buitrago L, Bhavanasi D, Dangelmaier C, Manne BK, Badolia R, Borgognone A, et al. Tyrosine phosphorylation on spleen tyrosine kinase (Syk) is differentially regulated in human and murine platelets by protein kinase $C$ isoforms. J Biol Chem. 2013;288(40):29160-9.

72. Unsworth AJ, Bombik I, Pinto-Fernandez A, McGouran JF, Konietzny R, Zahedi RP, et al. Human platelet protein Ubiquitylation and changes following GPVI activation. Thromb Haemost. 2019;119(1):104-16.

73. Kim S, Mangin P, Dangelmaier C, Lillian R, Jackson SP, Daniel JL, et al. Role of phosphoinositide 3-kinase beta in glycoprotein VI-mediated Akt activation in platelets. J Biol Chem. 2009;284(49):33763-72.

\section{Publisher's Note}

Springer Nature remains neutral with regard to jurisdictional claims in published maps and institutional affiliations.
Ready to submit your research? Choose BMC and benefit from:

- fast, convenient online submission

- thorough peer review by experienced researchers in your field

- rapid publication on acceptance

- support for research data, including large and complex data types

- gold Open Access which fosters wider collaboration and increased citations

- maximum visibility for your research: over $100 \mathrm{M}$ website views per year

At BMC, research is always in progress.

Learn more biomedcentral.com/submissions 